TRANSACTIONS OF THE

AMERICAN MATHEMATICAL SOCIETY

Volume 350, Number 6, June 1998, Pages 2229-2263

S 0002-9947(98)02109-6

\title{
TOPOLOGICAL CONDITIONS FOR THE EXISTENCE OF ABSORBING CANTOR SETS
}

\author{
HENK BRUIN
}

\begin{abstract}
This paper deals with strange attractors of S-unimodal maps $f$. It generalizes earlier results in the sense that very general topological conditions are given that either

i) guarantee the existence of an absorbing Cantor set provided the critical point of $f$ is sufficiently degenerate, or

ii) prohibit the existence of an absorbing Cantor set altogether.

As a by-product we obtain very weak topological conditions that imply the existence of an absolutely continuous invariant probability measure for $f$.
\end{abstract}

\section{INTRODUCTION}

Attractors are one of the central themes in dynamics, but a universal definition of attractor is hard to give. Already in the context of interval maps one encounters ambiguities that one would hardly expect. Let us illustrate this by the well-established classification of attractors for S-unimodal interval maps. First we need to distinguish between a metric and a topological attractor.

Definition (cf. [Mi1]). A closed set $A$ is a metric (topological) attractor, if

i) The basin $B(A)=\{x \mid \omega(x) \subset A\}$ has positive Lebesgue measure (is a residual set).

ii) There is no proper closed subset $A^{\prime} \subset A$ such that $B\left(A^{\prime}\right)$ has positive Lebesgue measure (is a residual set).

The following classification is due to Guckenheimer [G] for the topological part, and to Blokh and Lyubich [BL1] for the metric part.

Classification of Attractors. Let $f: I \rightarrow I$ be a non-flat S-unimodal (i.e. the Schwarzian derivative of $f$ is negative) map of the interval. Then $f$ has a unique topological attractor, which is one of the following:

i) An attracting periodic orbit.

ii) The union of $n$ intervals which are cyclically permuted by $f$. On each of these intervals $f^{n}$ is topological transitive.

iii) A Cantor set on which $f$ acts as an adding machine. This is the infinitely renormalizable case.

Furthermore, $f$ has a unique metric attractor, which can be of type i), ii), iii) or

Received by the editors June 1, 1995.

1991 Mathematics Subject Classification. Primary 58F13, 58F11.

Key words and phrases. Attractors, unimodal maps, invariant measures.

Supported by the Netherlands Organization for Scientific Research (NWO). The research for this paper was carried out during the author's stay at the University of Erlangen-Nürnberg.

(C)1998 American Mathematical Society 
iv) A Cantor set, but iii) does not apply: $f$ is finitely renormalizable.

An attractor of type iv) is called an absorbing Cantor set. It is a metric but not a topological attractor. It has been an open question for some years whether case iv) can occur. If $\ell=2$, absorbing Cantor sets do not exist. Proofs were given by Lyubich [L1] and by Jakobson and Świątek [JS1], [JS2], [JS3]. However, as was proved in [BKNS], there are maps (with a degenerate critical point) that have an absorbing Cantor set. This applies at least to maps with a special combinatorial structure, known as the Fibonacci dynamics.

The purpose of this paper is to generalize the results of [BKNS]: We want to indicate which topological constraints allow/prohibit the existence of absorbing Cantor sets.

The topology of absorbing Cantor sets is known to some extent [BL2], [GJ], [Ma1]. If $A$ is an absorbing Cantor set, then $A=\omega(c)$ (where $c$ is the critical point of $f), A$ is minimal and $c$ must have a rigid recurrence behaviour, sometimes called persistent recurrence. The precise formulation of this recurrence behaviour may change from one author to the next. In section 3 we will discuss this in detail: The differences in formulation are very subtle, leading to intricate counterexamples (section 11).

Let us discuss some of the ideas and tools used in this paper, and then state the main result in a simplified form.

Our main tool is a certain kind of induced map (section 4). It is a generalization of the induced map used in [BKNS]. The dynamics of the induced map $F$ can be considered as a random walk on a Markov chain with countably many states, $\left\{U_{k}\right\}_{k \in \mathbb{N}}$. From a topological viewpoint, $F$ is well-understood. The possible transitions from one state to another are given by the combinatorial structure of the original map $f$. If $f$ is not renormalizable, the set of dense orbits on the chain is residual in the set of all orbits. For the map $f$, this means that the set of points having a dense $f$-orbit is also residual.

From a measure theoretical viewpoint, one has to distinguish between recurrent and transient Markov chains. In principle, if a point $x$ escapes to infinity under iteration of $F$, then $x$ tends to $\omega(c)$ under iteration of $f$. Therefore a transient Markov chain corresponds to a map with an absorbing Cantor set (section 5). We will have to compute the probabilities of going from one state to another. This involves complicated estimates, which makes up the hardest part of the proofs.

We will describe the combinatorics of a unimodal map by means of the kneading map $Q$. This map has been developed by Hofbauer and Keller, e.g. [H], [HK]. It is a map on $\mathbb{N}$ with the property that $Q(n)<n$ for all $n>0$. For example, the Fibonacci map has the kneading map $Q(k)=\max \{k-2,0\}$. The precise definition and some of the properties of $Q$ will be given in section 2. Here we will point out how $Q$ describes the dynamics on the Markov chain: From state $U_{k}$ one can reach $U_{l}$ if and only if

$$
l \geq \min \{Q(k)+1, Q(Q(k-1)+1)+1\} .
$$

Therefore if $k-Q(k)$ is bounded ( $f$ is Fibonacci-like), then one can drop only a bounded number of states in the Markov chain. This gives good chances to find a transient Markov chain. We conjecture that the boundedness of $k-Q(k)$ is sufficient for the existence of an absorbing Cantor set (provided the critical order $\ell$ is sufficiently large). Using an additional condition on $Q$, we can indeed prove 
this conjecture (section 6). On the other hand, we can show that in general no absorbing Cantor set can arise if $k-Q(k) \rightarrow \infty$, irrespective the value of $\ell$ (section 8).

Related to the question of absorbing Cantor sets is the question whether $f$ has an absolutely continuous (with respect to Lebesgue measure) invariant probability measure (acip for short). As was proved in $[\mathrm{KN}],[\mathrm{LM}]$, the Fibonacci map has an acip if the order $\ell$ of the critical point is sufficiently small $(\ell<2+\varepsilon)$. It is conjectured that the picture is as follows: Let $f_{\ell}$ be a family of Fibonacci maps with critical order $\ell$. For $\ell$ small, $f_{\ell}$ has an acip. If $\ell$ increases, the acip disappears, to be replaced by a conservative absolutely continuous infinite $\sigma$-finite invariant measure. If $\ell$ increases even more, then an absorbing Cantor set is created, and $f_{\ell}$ is therefore no longer conservative.

The same picture may be true for Fibonacci-like maps. However, using the summability condition of Nowicki and van Strien [NS], we can give a large class of combinatorial structures that imply the existence of an acip, irrespective the value of $\ell$ (section 9). In this case the stage of a $\sigma$-finite measure is never reached.

Let us summarize this exposition in the following, simplified, theorem:

Theorem A. Let $f$ be a finitely renormalizable, non-flat $S$-unimodal map having critical order $\ell<\infty$ and kneading map $Q$. Assume that $Q$ is eventually nondecreasing.

a) If $k-Q(k)$ is bounded, then there exists $\ell_{0}$ (depending only on the upper bound of $k-Q(k)$ ) such that $f$ has an absorbing Cantor set, whenever $\ell>\ell_{0}$.

b) If $\lim k-Q(k)=\infty$, then $f$ has no absorbing Cantor set.

c) If $\lim \frac{k-Q(k)}{\log k}=\infty$, then $f$ has an absolutely continuous invariant probability measure.

The assumption that $Q$ is eventually non-decreasing is strong, but it simplifies the metric estimates considerably. Among other things, these metric estimates are needed to show that certain (dynamically defined) points $u_{k}$ accumulate exponentially fast on $c$. In section 7 (and 10), we derive sufficient topological conditions for this behaviour. These conditions are much weaker than that $Q$ is eventually non-decreasing. In fact, Theorem A is a special case of the Theorems 6.1, 8.1 and 9.1 .

We want to thank Gerhard Keller for many fruitful discussions. We also thank the referee for useful remarks.

\section{Notation And Preliminaries}

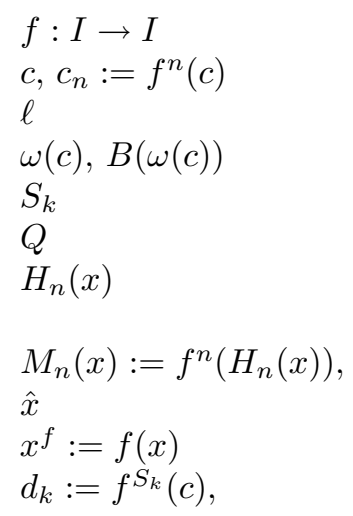

$S$ - unimodal map on the unit interval, critical point and its iterates, order of the critical point, critical omega-limit set and its basin of attraction, $k$-th cutting time, kneading map, maximal interval of monotonicity of $f^{n}$ containing $x$, involution of $x: f(\hat{x})=f(x)$, used for points close to $c_{1}$, 


$$
\begin{aligned}
& z_{k}, A_{k}:=\left(z_{k-1}, z_{k}\right) \\
& u_{k}, U_{k}:=\left(u_{k-1}, u_{k}\right) \\
& F \\
& F \mid U_{k} \cup \hat{U}_{k}:=f^{S_{k-1} \mid U_{k} \cup \hat{U}_{k},} \\
& \varphi_{n}(x) \\
& y_{k}:=f^{S_{Q(k)-1}}\left(d_{k-1}\right), \\
& t_{k}^{f}
\end{aligned}
$$

$k$-th closest precritical point, generalized closest prefixed points, induced map,

$\varphi_{n}(x)=k$ if and only if $F^{n}(x) \in U_{k}$,

right boundary point of $H_{S_{k}-1}\left(c_{1}\right)$.

In more detail:

- $f:[0,1] \rightarrow[0,1]$ is a unimodal map with $f(0)=f(1)=0$. We assume that $f$ is $C^{3}$ and has negative Schwarzian derivative $\left(S f:=\frac{D^{3} f}{D f}-\frac{3}{2}\left(\frac{D^{2} f}{D f}\right)^{2}<0\right.$ wherever it is defined).

- $f$ is called renormalizable of period $n>1$ if there is an interval $c \in J \subsetneq$ $\left[c_{2}, c_{1}\right]$ such that $f^{n}(J) \subset J$. The maximal interval with this property is called restrictive. We assume throughout the paper that $f$ admits no periodic attractor and is not renormalizable.

- || denotes Lebesque measure or just the absolute value. $d(A, B)$ is the distance between sets or points.

- The order of the critical point $\ell<\infty$. Hence $|f(c)-f(x)|=\mathcal{O}(1)|c-x|^{\ell}$ and $D f(x)=\mathcal{O}(\ell)|x-c|^{\ell-1}$. It follows that the involution $x \mapsto \hat{x}$ ( $\hat{x}$ is such that $\hat{x} \neq x$ (if $x \neq c$ ) and $f(\hat{x})=f(x))$ is Lipschitz. For simplicity we will assume that $\hat{x}=2 c-x$.

- $\omega(x)$ is the set of accumulation points of $\operatorname{orb}(x)$, the forward orbit of $x$. A forward invariant set $A$ is minimal if every $x \in A$ has a dense orbit in $A$.

- $\omega(x)$ is minimal if and only if $x$ is uniformly recurrent [Got], i.e. for every neighbourhood $U$ of $x$, there exists $N=N(U)$ such that for every $m$ for which $f^{m}(x) \in U$, there exists $n \leq N$ such that also $f^{m+n}(x) \in U$.

- Suppose that $H_{n}(x)=(a, b) \ni x$ is the maximal interval on which $f^{n}$ is diffeomorphic; then

$$
r_{n}(x):=\min \left\{\left|f^{n}(x)-f^{n}(a)\right|,\left|f^{n}(x)-f^{n}(b)\right|\right\},
$$

and

$$
R_{n}(x):=\max \left\{\left|f^{n}(x)-f^{n}(a)\right|,\left|f^{n}(x)-f^{n}(b)\right|\right\} .
$$

Contraction Principle. For every $\varepsilon>0$ there exists $\delta>0$ such that for every $n \geq 0$ and every interval of size $|J|>\varepsilon,\left|f^{n}(J)\right| \geq \delta$.

In particular, $f^{n} \mid J$ cannot be homeomorphic for every $n$. The contraction principle holds if there are no wandering intervals or periodic attractors [BL2], [MS].

Let $g: J \rightarrow \mathbb{R}$ be $C^{1}$; then the distortion

$$
\operatorname{dis}(g, J):=\sup _{x, y \in J} \frac{|D g(x)|}{|D g(y)|} .
$$

If $g$ has negative Schwarzian derivative, $g$ expands the cross ratio: Let $j \subset t$ be intervals, and let $l, r$ be the components of $t \backslash j$. Then $\frac{|t||j|}{|l||r|}$ is the cross-ratio of $j$ and $t$. 


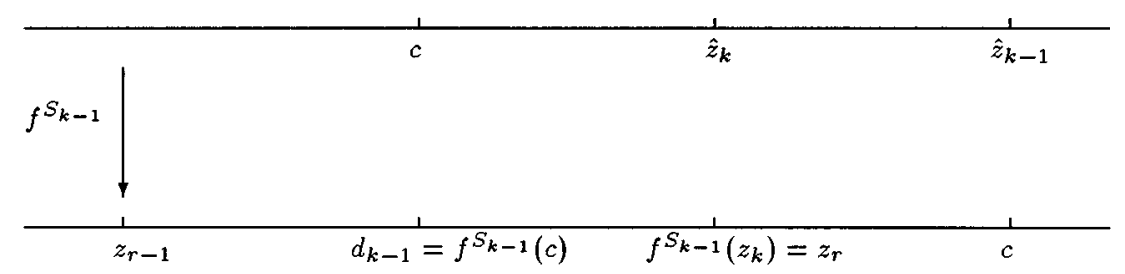

Figure 2.1

Expansion of Cross-Ratios. Let $g: t \rightarrow T$ be a diffeomorphism with negative Schwarzian derivative. Then $g$ expands the cross-ratio, i.e.

$$
\frac{|t||j|}{|l||r|} \leq \frac{|T||J|}{|L||R|}
$$

where the capital letters indicate the g-images.

Furthermore, we can use the Koebe principle (see e.g. [MS]). Let $T \supset J$ again be intervals. $T$ is said to contain a $\delta$-scaled neighbourhood of $J$ if both components of $T \backslash J$ have size $\geq \delta|J|$.

Koebe Principle. Suppose $S g \leq 0$. Fix $\delta>0$ and let $K=\left(\frac{1+\delta}{\delta}\right)^{2}$. Then the following property holds: Let $j \subset t$ be intervals such that $g \mid t$ is monotone. If $T$ contains a $\delta$-scaled neighbourhood of $J$, then $\operatorname{dis}(g, j) \leq K$.

A forward iterate $c_{n}$ is called a closest return if $c_{j} \notin\left[c_{n}, \hat{c}_{n}\right]$ for $0<j<n$. The closest precritical points $z_{k}$ and cutting times $S_{k}$ are defined as follows: $S_{0}:=1$, $z_{0}:=f^{-1}(c) \cap(0, c)$. Inductively,

$$
S_{k+1}:=\min \left\{n>S_{k} \mid f^{-n}(c) \cap\left(z_{k}, c\right)\right\}
$$

and

$$
z_{k+1}:=f^{-S_{k+1}}(c) \cap\left(z_{k}, c\right)
$$

We will give a few properties of these notions. More details can be found in [B2], [B3]. Let $A_{k}:=\left(z_{k-1}, z_{k}\right)$, and $A_{0}:=\left(0, z_{0}\right)$. If $S_{k}<n \leq S_{k+1}$, then $\left(z_{k}, c\right)$ and $\left(c, \hat{z}_{k}\right)$ are maximal intervals on which $f^{n}$ is diffeomorphic.

By construction, $f^{S_{k-1}}\left(z_{k}\right)$ is again a closest precritical point; see Figure 2.1. The kneading map $Q: \mathbb{N} \rightarrow \mathbb{N}$ is defined so that

$$
f^{S_{k-1}}\left(z_{k}\right)=z_{Q(k)} \text {. }
$$

It follows that

$$
S_{k}=S_{k-1}+S_{Q(k)}
$$

The kneading map determines the combinatorics of the map completely. Define $d_{k}:=f^{S_{k}}(c)$. By Figure 2.1 and the construction of the closest preimages,

$$
d_{k-1} \in\left(z_{Q(k)-1}, z_{Q(k)}\right) \cup\left(\hat{z}_{Q(k)}, \hat{z}_{Q(k)-1}\right) .
$$

This is true for all $k \geq 1$. If $Q(k)=0$, then $d_{k-1} \in I \backslash\left(z_{0}, \hat{z}_{0}\right)$. Notice also that $f^{S_{k}}$ maps $\left(z_{k-1}, c\right)$ diffeomorphically onto $\left(d_{Q(k)}, d_{k}\right)$ and $f^{S_{k}}\left(z_{k}\right)=c$. Therefore $d_{k}$ and $d_{Q(k)}$ lie on different sides of $c$.

Lemma 2.1. If there is no periodic attractor, then $Q(k)<k$ for all $k \geq 1$. 


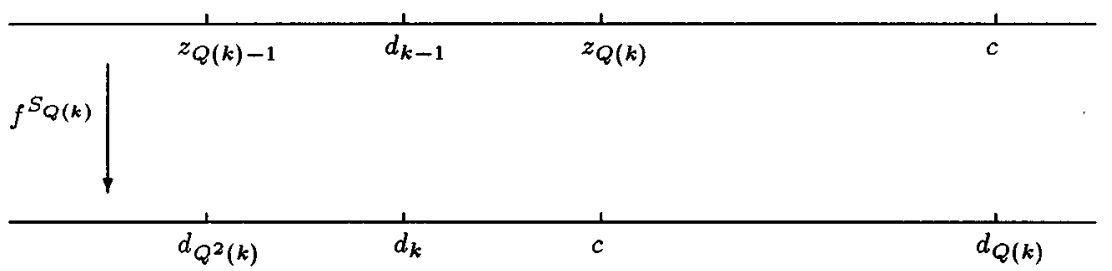

FiguRE 2.2

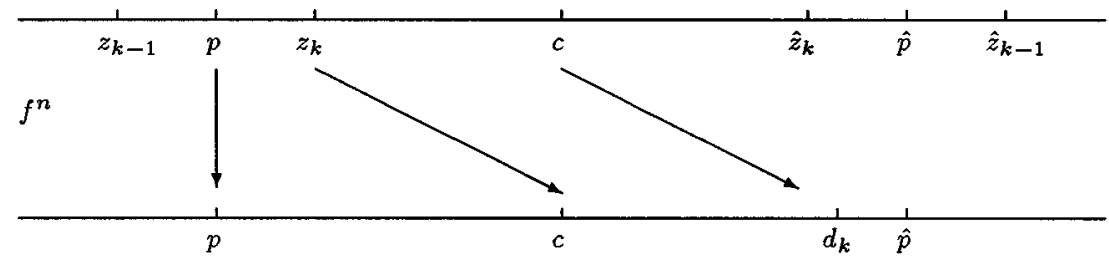

FiguRE 2.3

Proof. $f^{S_{k-1}}$ maps both $\left(z_{k-1}, c\right)$ and $\left(c, \hat{z}_{k-1}\right)$ diffeomorphically onto $\left(d_{k-1}, c\right)$. If $Q(k) \geq k$, then by $(2.2), d_{k} \in\left(z_{k-1}, \hat{z}_{k-1}\right)$. Hence $f^{S_{k-1}}$ maps either $\left(z_{k-1}, c\right)$ or $\left(c, \hat{z}_{k-1}\right)$ diffeomorphically into itself, yielding a periodic attractor.

Lemma 2.2. There exists a unimodal map with $Q$ as kneading map if and only if

$$
\{Q(k+j)\}_{j \geq 1} \succeq\left\{Q\left(Q^{2}(k)+j\right)\right\}_{j \geq 1},
$$

where $\succeq$ denotes lexicographical order.

Proof. See $[\mathrm{H}]$ or [B3]. Formula (2.3) is the admissibility condition for kneading maps. The geometric interpretation is that $\left|d_{k}-c\right| \leq\left|d_{Q^{2}(k)}-c\right|$ for all $k$.

This follows immediately from (2.2) by taking the $S_{Q(k)}$-th iterate. See Figure 2.2 .

Lemma 2.3. If $f$ is renormalizable of period $n$, but has no n-periodic attractor, then there exists $k$ such that $S_{k}=n$ and $Q(k+j) \geq k$ for all $j \geq 1$.

Proof. Let $[p, \hat{p}]$ be the restrictive interval of period $n$. Then the situation is as in Figure 2.3.

Because $f^{n}((p, c)) \ni c$ and $f^{n} \mid(p, c)$ is diffeomorphic, $(p, c) \ni z_{k}$ for some $k$ minimal, and $n=S_{k}$. Therefore $p \in\left(z_{k-1}, z_{k}\right)$. By Lemma 2.1, $d_{k} \notin\left(z_{k}, \hat{z}_{k}\right)$, and as $d_{k} \in[p, \hat{p}], d_{k} \in\left(\hat{z}_{k}, \hat{z}_{k-1}\right)$. Because $f^{j}([p, \hat{p}]) \ni c$ only if $j$ is a multiple of $n=S_{k}$ (in which case $\left.f^{j}([p, \hat{p}]) \subset[p, \hat{p}]\right), d_{m} \in[p, \hat{p}] \subset\left(z_{k-1}, \hat{z}_{k-1}\right)$ for every $m \geq k$. Therefore $Q(m+1) \geq k$ for every $m \geq k$.

We will often have to impose the following condition on the combinatorics:

$$
Q(k+1)>Q\left(Q^{2}(k)+1\right) \text { for all sufficiently large } k \text {. }
$$

Geometrically, this means that there is at least one closest precritical point between $d_{k}$ and $d_{Q^{2}(k)}$ (see Figure 2.2). We have not been able to prove most of the metric estimates without (2.4) or a similar condition. Condition (2.4) prohibits the existence of saddle-node like returns (see Section 7). Clearly (2.4) is true if $Q$ is eventually non-decreasing. Moreover, 


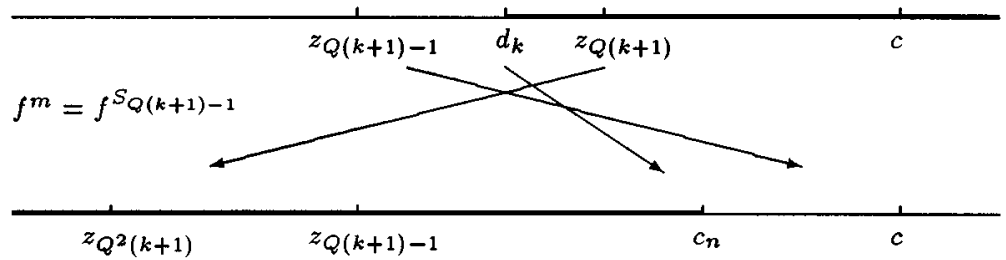

FiguRe 2.4

Lemma 2.4. If $Q$ is eventually non-decreasing and $Q(k) \rightarrow \infty$, then there exists $k_{0}$ such that for all $k \geq k_{0}, d_{k}$ is a closest return and there is no other closest return past $d_{k_{0}}$.

Proof. Suppose that $k_{0}$ is such that $Q(k+1) \geq Q(k)$ for all $k \geq k_{0}$, and take $k_{0}$ such that $Q(k)>k_{1}$ for all $k \geq k_{0}$.

We will first show that $d_{k+1} \in\left(d_{k}, \hat{d}_{k}\right)$ for all $k \geq k_{0}$. Suppose by contradiction that $d_{k} \in\left(d_{k+1}, \hat{d}_{k+1}\right)$ for some $k \geq k_{0}$. Then $Q(k+2) \leq Q(k+1)$, so in fact $Q(k+2)=Q(k+1)=n$. By $(2.2), d_{k} \in\left(d_{k+1}, z_{n}\right) \cup\left(\hat{z}_{n}, \hat{d}_{k+1}\right)$. As $d_{k+2}=$ $f^{S_{n}}\left(d_{k+1}\right), d_{k+1}=f^{S_{n}}\left(d_{k}\right)$ and $f^{S_{n}}\left(z_{n}\right)=c, d_{k+1} \in\left(d_{k+2}, c\right)$. It follows by induction that $Q(k+j+1)=Q(k+j)$ for all $j \geq 1$, contradicting our assumption that $Q(k) \rightarrow \infty$.

Next suppose that $c_{n}$ is a closest return for some $S_{k}<n<S_{k+1}$. Without loss of generality we can assume that $c_{n} \in\left(d_{k}, c\right)$ and $d_{k} \in\left(z_{Q(k+1)-1}, z_{Q(k+1)}\right)$. Write $m:=n-S_{k}<S_{Q(k+1)}$. As $c_{n}$ is a closest return, $M_{n-1}\left(c_{1}\right) \ni c$. Hence also $M_{m}\left(d_{k}\right) \ni c$ and $H_{m}\left(d_{k}\right)$ contains a preimage $x$ of $c$. As there are no points of $f^{-m}(c)$ in $\left(z_{Q(k+1)-1}, c\right), f^{m}\left(z_{Q(k+1)-1}\right) \in\left[c, c_{n}\right]$. Because $z_{Q(k+1)-1}$ is a closest precritical point, in fact $f^{m}\left(z_{Q(k+1)-1}\right)=c$, so $m=S_{Q(k+1)-1}$. It follows that $z_{Q(k+1)-1} \in f^{m}\left(\left[d_{k}, z_{Q(k+1)}\right]\right)=\left[z_{Q^{2}(k+1)}, c_{n}\right]$. See Figure 2.4. Therefore $r:=$ $n+S_{Q(k+1)-1}$ is a cutting time. Furthermore,

$$
\begin{aligned}
r & =S_{k}+2 S_{Q(k+1)-1}=S_{k+1}+S_{Q(k+1)-1}-S_{Q^{2}(k+1)} \\
& <S_{k+1}+S_{Q(k+2)}=S_{k+2} .
\end{aligned}
$$

Hence $r=S_{k+1}$. But then $Q(Q(k+1))=Q(k+1)-1$, and because $Q$ is nondecreasing, $f$ is renormalizable with period $S_{Q(k+1)-1}$. Passing to the renormalization (which has a non-decreasing kneading map) and repeating the arguments, we get the result.

Let $t_{k}^{f}$ be such that

$$
H_{S_{k}-1}\left(c^{f}\right)=\left(z_{k-1}^{f}, t_{k}^{f}\right) .
$$

Notice that $t_{k}^{f} \notin\left[c_{2}, c_{1}\right]$, so $t_{k}^{f}$ is not the image of any point $t_{k}$. The notation is just to indicate that $t_{k}^{f}$ is close to $c^{f}$.

Lemma 2.5. Let $\kappa:=\min \{k \mid Q(k)>0\}$. Then for all $k>\kappa, M_{S_{k}-1}\left(c^{f}\right)=$ $f^{S_{k}-1}\left(z_{k-1}^{f}, t_{k}^{f}\right) \subset\left(d_{Q(k)}, d_{Q^{2}(k)}\right)$. If (2.4) holds, then $M_{S_{k}-1}\left(c^{f}\right)=\left(d_{Q(k)}, d_{Q^{2}(k)}\right)$, for $k$ sufficiently large.

Proof. By (2.1), at least $f^{S_{k}-1}\left(z_{k-1}^{f}\right)=d_{Q(k)}$. For $k=\kappa+1, f^{S_{k}-1}\left(t_{k}^{f}\right)=c_{1}=$ $d_{Q^{2}(k)}$, as one can verify by hand. Now take $k>\kappa+1$ arbitrary and suppose 


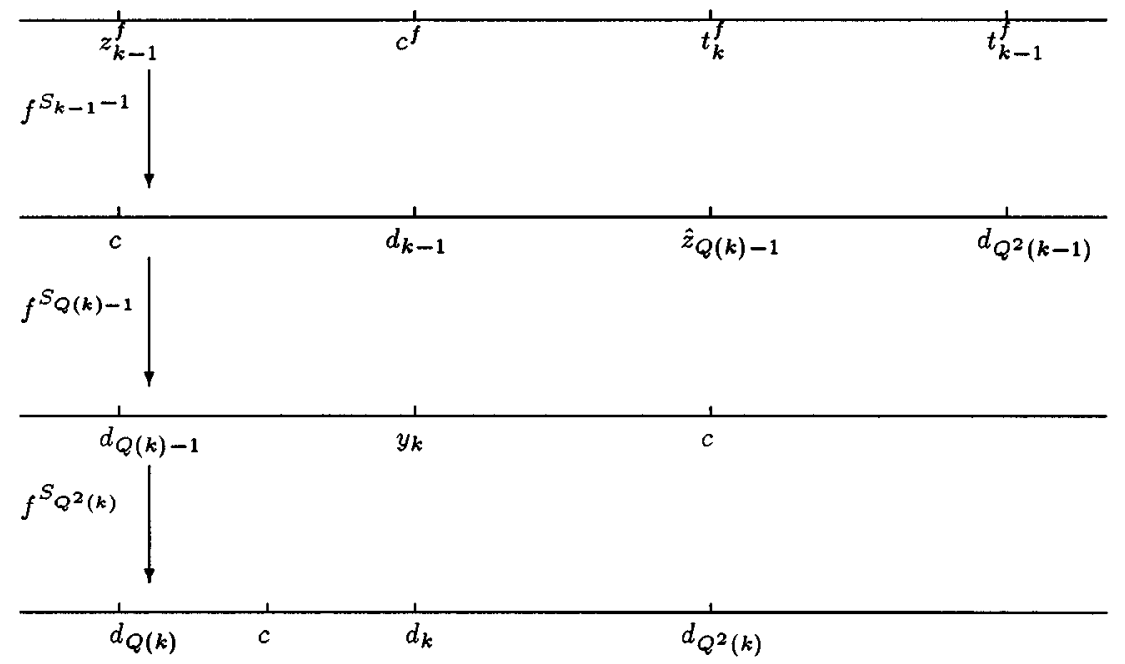

FiguRE 2.5

without loss of generality that $d_{k-1}>c$. Therefore by $(2.2), d_{k-1} \in\left(c, \hat{z}_{Q(k)-1}\right)$. If

$$
\hat{z}_{Q(k)-1} \in\left(d_{k-1}, f^{S_{k-1}-1}\left(t_{k-1}^{f}\right)\right),
$$

then by construction of $H_{S_{k}-1}\left(c^{f}\right), f^{S_{k-1}-1}\left(t_{k}^{f}\right)=\hat{z}_{Q(k)-1}$. Hence $f^{S_{k}-1}\left(t_{k}^{f}\right)=$ $f^{S_{Q(k)}}\left(\hat{z}_{Q(k)-1}\right)=d_{Q^{2}(k)}$. If $(2.5)$ is false, so $f^{S_{k-1}-1}\left(t_{k-1}^{f}\right) \in\left(d_{k-1}, \hat{z}_{Q(k)-1}\right)$, then $t_{k}^{f}=t_{k-1}^{f}$ and $f^{S_{k}-1}\left(t_{k}^{f}\right) \in f^{S_{Q(k)}}\left(\left(d_{k-1}, \hat{z}_{Q(k)-1}\right)\right)=\left(d_{k}, d_{Q^{2}(k)}\right)$. This proves the first statement. If $f^{S_{k-1}-1}\left(t_{k-1}^{f}\right)=d_{Q^{2}(k-1)}$ and $Q\left(Q^{2}(k-1)+1\right)<Q(k)$, then (2.5) is satisfied. In this case all the relations of Figure 2.5 (with $y_{k}:=f^{S_{Q(k)-1}}\left(d_{k-1}\right)$ ) are true. By induction the second statement follows too.

For the proofs of the following statements, we refer to [Ma2].

- A point $x$ is said to be nice if its forward orbit does not enter $(x, \hat{x})$.

- An interval is called nice if it is symmetric and has nice boundary points.

- Let $V$ be a nice interval and let $m>0$ be the smallest integer such that $c_{m} \in$ $V$. Then because $f$ is not renormalizable, $f^{m}(V)$ contains a boundary point $x$ of $V$ in its interior. Pulling back this point along the orbit $c, c_{1}, \ldots, c_{m}$ we obtain two points $y$ and $\hat{y}$ in $V . y$ and $\hat{y}$ are nice. Define $\psi$ by $\psi(V)=(y, \hat{y})$.

- Let $V$ be a nice interval and $x \notin V$. If $n$ is the first visit time of $x$ to $V$, then $M_{n}(x) \supset V$.

- Additionally, let $x \notin \psi(V)$, and $n$ be the first visit time of $x$ to $\psi(V)$. Then $M_{n}(x) \supset V$.

\section{Persistent Recurrence and Related Notions}

In this section we discuss several notions concerning the recurrence behaviour of the critical point:

- Johnson and Guckenheimer [GJ] introduced critical monotonicity as a sufficient condition for the NON-existence of absorbing Cantor sets.

- Blokh and Lyubich [BL2] gave a necessary topological condition $\left(r_{n}(x) \rightarrow 0\right.$ for $x \in \omega(c))$ for the existence of absorbing Cantor sets. 
- In complex dynamics the notion of persistent recurrence has been used: it refers to Yoccoz' $\tau$-function. Real interpretations of persistent recurrence appear in papers of Lyubich [L1]-[L2].

- The existence of absorbing Cantor sets was proven for sufficiently flat Fibonacci maps. In this paper we show that absorbing Cantor sets can also be found for more general, Fibonacci-like, maps.

It is not clear from the start which condition implies which, and in fact the difference is very subtle in some cases. In this section we want to classify the several versions of recurrence and discuss their relations (knowing that some of these relations were proved in [BL2], [L1], [Mi2]).

(C1) $f$ is Fibonacci-like, i.e. $k-Q(k)$ is bounded.

(C2) $Q(k) \rightarrow \infty$.

(C3) $R_{n}\left(c_{1}\right) \rightarrow 0$.

(C4) For every neighbourhood $U \ni c$ there exists $N$ such that for every $n>N$, $M_{n}\left(c_{1}\right)$ does not cover a component of $U \backslash\{c\}$.

(C5) For every symmetric neighbourhood $U \ni c$ there exists $N$ such that for every $n>N, M_{n}\left(c_{1}\right) \not \supset U$

(C6) For every symmetric neighbourhood $U \ni c$ there exists $N$ such that for every $n>N: c_{n+1} \notin U$ or $M_{n}\left(c_{1}\right) \not \supset U$ (i.e. $f$ is NOT critically monotonic).

(C7) For every nice interval $U \ni c$, there exists $N$ such that for every $n>N$ : $c_{n+1} \notin U$ or $M_{n}\left(c_{1}\right) \not \supset U$.

(C8) $r_{n}\left(c_{1}\right) \rightarrow 0$.

(C9) $\omega(c)$ is a minimal Cantor set containing $c$.

Proposition 3.1. The following implications hold:

$$
(C 1) \Rightarrow(C 2) \Leftrightarrow(C 3) \Leftrightarrow(C 4) \Rightarrow(C 5) \Rightarrow(C 6) \Rightarrow(C 7) \Leftrightarrow(C 8) \Rightarrow(C 9)
$$

Here $\Rightarrow$ also means that the reverse implication is false.

Proof. $(\mathrm{C} 1) \Rightarrow(\mathrm{C} 2)$ : Trivial.

$(\mathrm{C} 2) \nRightarrow(\mathrm{C} 1)$ : Trivial.

$(\mathrm{C} 2) \Rightarrow(\mathrm{C} 3)$ : Lemma 2.5 states that $M_{S_{k}-1}\left(c_{1}\right) \subset\left(d_{Q(k)}, d_{Q^{2}(k)}\right)$. If $Q(k) \rightarrow \infty$, then $R_{S_{k}-1}\left(c_{1}\right)<\left|d_{Q(k)}-d_{Q^{2}(k)}\right|<\left|z_{Q(Q(k)+1)-1}-\hat{z}_{Q\left(Q^{2}(k)+1\right)-1}\right| \rightarrow 0$ as $k \rightarrow \infty$. By the Contraction Principle, also $R_{n}\left(c_{1}\right) \rightarrow 0$.

$(\mathrm{C} 3) \Rightarrow(\mathrm{C} 2) \lim _{n} R_{n}\left(c_{1}\right) \rightarrow 0$ implies $\lim _{k} R_{S_{k}-1}\left(c_{1}\right) \rightarrow 0$, whence $\left|d_{k}-c\right| \rightarrow 0$. As $\left(d_{k}, c\right) \ni z_{Q(k+1)}$ or $\hat{z}_{Q(k+1)}, Q(k) \rightarrow \infty$.

(C3) $\Rightarrow(\mathrm{C} 4)$ : Trivial.

$(\mathrm{C} 4) \Rightarrow(\mathrm{C} 3)$ : Assume by contradiction that $\lim \inf R_{n}\left(c_{1}\right) \geq \varepsilon>0$. Then there exist arbitrarily large integers $n$ such that $\left|M_{n}\left(c_{1}\right)\right| \geq \varepsilon$. Due to the Contraction Principle, there exist $\delta>0$ and arbitrarily large integers $m$ such that $\left|M_{m}\left(c_{1}\right)\right|>\delta$ and $M_{m}\left(c_{1}\right) \ni c$. Therefore $M_{m}\left(c_{1}\right)$ contains a component of $(c-\delta / 2, c+\delta / 2) \backslash\{c\}$.

$(\mathrm{C} 4) \Rightarrow(\mathrm{C} 5)$ : Trivial.

$(\mathrm{C} 5) \nRightarrow(\mathrm{C} 4)$ : See Example 11.2.

$(\mathrm{C} 5) \Rightarrow(\mathrm{C} 6)$ : Trivial.

$(\mathrm{C} 6) \nRightarrow(\mathrm{C} 5)$ : See Example 11.3.

$(\mathrm{C} 6) \Rightarrow(\mathrm{C} 7)$ : Trivial.

$(\mathrm{C} 7) \nRightarrow(\mathrm{C} 6)$ See Example 11.1.

$(\mathrm{C} 7) \Rightarrow(\mathrm{C} 8)$ : Assume $(\mathrm{C} 7)$ holds but $\lim \sup r_{n}\left(c_{1}\right)=\delta>0$. Choose a nice interval $V$ so small that according to the Contraction Principle no interval of length 
$\geq 2 \delta$ can be mapped in a monotonic way into $V$. Let $n$ be arbitrary such that $r_{n}\left(c_{1}\right) \geq \delta$. Let $m \geq n$ be the smallest iterate such that $c_{m+1} \in V$. As $V$ is nice, $M_{m-n}\left(c_{n+1}\right) \supset V$, and by the choice of $V, H_{m-n}\left(c_{n+1}\right) \cap f^{n-m}(V) \subset M_{n}\left(c_{1}\right)$. Therefore also $M_{m}\left(c_{1}\right) \supset V$. Since this can be done for infinitely many $n,(\mathrm{C} 7)$ is false after all.

$(\mathrm{C} 8) \Rightarrow(\mathrm{C} 9):$ As $r_{n}\left(c_{1}\right) \rightarrow 0, c$ is recurrent. Recall that $f$ is assumed to have no periodic attractor, so \# $(\operatorname{orb}(c))=\infty$. Assume by contradiction that $\omega(c)$ is not minimal. Then $c$ is not uniformly recurrent. Therefore there exists a set $V \ni c$ such that $b(n):=\min \left\{k-n \mid k>n, c_{k} \in V\right\}$ can be arbitrarily large. Without loss of generality assume that $V$ is nice. If $c_{n} \in V$, then we can pull $V$ back along the orbit $c_{n}, c_{n+1}, \ldots, c_{n+b(n)}$, obtaining an interval $V_{n} \subset V$. As $V$ is nice, $V_{n} \cap V_{m}=\emptyset$ if $b(n) \neq b(m)$. (In particular, $V_{0}$ is the only interval of this kind on which $f^{b(n)}=f^{b(0)}$ is not monotone.) Because $b(n)$ can be arbitrarily large, there are infinitely many sets $V_{n}$.

Next take $m(n)=\min \left\{k \geq 1 \mid c_{k} \in V_{n}\right\}$. Then for each $V_{n}$, there exists a set $V_{n}^{\prime} \ni c_{1}$ such that $f^{m(n)-1}$ maps $V_{n}^{\prime}$ monotonically onto $V_{n}$.

Now let $U:=\psi(V)$. As $f$ is not renormalizable, $U \neq V$. Let $\delta$ be the minimum length of the components of $V \backslash U$. Let $m^{\prime}(n):=\min \left\{k \geq 0 \mid c_{m(n)+k} \in U\right\}$. Then there exists $V_{n}^{\prime \prime} \supset c_{1}$ such that $f^{m^{\prime}(n)+m(n)-1}$ maps $V_{n}^{\prime \prime}$ monotonically onto $V$, with $c_{m^{\prime}(n)+m(n)} \in U$. Therefore $r_{m^{\prime}(n)+m(n)-1}\left(c_{1}\right) \geq \delta$. Because this is true infinitely often, $r_{k}\left(c_{1}\right) \not 0$.

$(\mathrm{C} 9) \nRightarrow(\mathrm{C} 8)$ : Counter-examples appear for example in [GT].

$(\mathrm{C} 8) \Rightarrow(\mathrm{C} 7)$ : Suppose $(\mathrm{C} 8)$ is true, and, by contradiction, that $(\mathrm{C} 7)$ is false. Therefore there exists a sequence of iterates $n_{i}$ such that $M_{n_{i}}\left(c_{1}\right) \supset V \ni c_{n_{i}+1}$, where $V$ is a nice interval. Because (C8) is true, $d\left(c_{n_{i}+1}, \partial V\right) \rightarrow 0$ as $i \rightarrow \infty$. This means that $\partial V \cap \omega(c) \neq \emptyset$, and $\omega(c)$ is not minimal. This contradicts the previous $(\mathrm{C} 8) \Rightarrow(\mathrm{C} 9)$ implication.

The notion of persistent recurrence comes from complex dynamics: A quadratic map $f(z)=z^{2}+c_{1}$ has a persistently recurrent critical point if $\tau(k) \rightarrow \infty$. Here $\tau$ is Yoccoz' $\tau$-function in the critical tableau. In order to relate this property to the ones we already have, we will assume in the next lemma that $f(z)=z^{2}+c_{1}$, $c_{1} \in \mathbb{R}$, instead of just a smooth unimodal map. Because of the notation $c_{1}$, we can maintain $c_{n}$ as the $n$-th iterate of the critical point.

Lemma 3.2. $c$ is persistently recurrent if and only if $r_{n}\left(c_{1}\right) \rightarrow 0$.

Proof. We will use the notation from [Mi2]. $P_{n}(x)$ denotes the order $n$ puzzle piece containing $x$.

First recall that the two boundary points of $P_{n}(c) \cap \mathbb{R}$ are preimages of the orientation-reversing fixed point $p$. They are symmetric to each other, and nice. In other words $P_{n}(c) \cap \mathbb{R}$ is a nice interval for each $n$.

Because of Proposition 3.1, it suffices to show that persistent recurrence is equivalent to property $(\mathrm{C} 7)$. Assume by contradiction that $c$ is not persistently recurrent. Therefore $\liminf \operatorname{in}_{n} \tau(n)=k<\infty$. Take $n$ arbitrary such that $f^{n-k}$ maps $P_{n}(c)$ by a two-fold covering onto $P_{k}(c)$. Then $f^{n-k-1}$ maps $f\left(P_{n}(c)\right) \cap \mathbb{R}$ to a fixed nice interval $P_{k}(c) \cap \mathbb{R} \ni c_{n-k}$. Therefore $(\mathrm{C} 7)$ is false.

Conversely, assume that $(\mathrm{C} 7)$ is false, and that $V$ is a nice interval such that $M_{n}\left(c_{1}\right) \supset V \ni c_{n+1}$ for arbitrarily large $n$. Take $k$ minimal such that $P_{k}(c) \cap \mathbb{R} \subset V$. For $n$ as above, let $m \geq 0$ be the smallest integer such that $c_{m+n+1} \in P_{k}(c) \cap \mathbb{R}$. 
It follows that $f^{n+m+1}$ maps $P_{n+m+1}(c)$ by a two-fold covering onto $P_{k}(c) \cap \mathbb{R}$. Hence $\tau(n+m+1)=k$ for arbitrarily large numbers $n$, and $c$ is not persistently recurrent.

Lemma 3.3. We have $r_{n}\left(c_{1}\right) \rightarrow 0$ if and only if $r_{n}(x) \rightarrow 0$ for every $x \in \omega(c)$, in which case $r_{n}(x) \rightarrow 0$ uniformly.

Proof. $\Leftarrow$ Trivial. Notice that it immediately follows that $\omega(c)$ is minimal. Indeed, if for some $x \in \omega(c), c \notin \omega(x)$, then $r_{n}(x) \nrightarrow 0$.

$\Rightarrow \quad$ Assume that there exists $\left\{x_{n}\right\} \subset \omega(c)$ such that $\limsup _{n} r_{n}\left(x_{n}\right) \geq \delta>0$. Choose $c \in U=\psi(V) \subsetneq V$ so small that for every interval $J$ of length $\geq 2 \delta$, $f^{n}(J) \not \subset V$ for every $n \geq 0$. We already know that $\omega(c)$ is minimal, so $c \in \omega\left(x_{n}\right)$. Choose $n$ arbitrarily large such that $r_{n}\left(x_{n}\right) \geq \delta$, and set $m_{1}(n):=\min \{k \geq n \mid$ $\left.f^{k}\left(x_{n}\right) \in V\right\}$. Pull $V$ back along the orbit $x_{n}, f\left(x_{n}\right), \ldots, f^{m_{1}(n)}\left(x_{n}\right)$, obtaining a neighbourhood of $V_{n}$ of $x_{n}$.

Let $m_{2}(n):=\min \left\{k \geq 1 \mid c_{k} \in V_{n}\right\}$. This gives an interval $V_{n}^{\prime} \ni c_{1}$ which is mapped monotonically onto $V$ by $f^{m_{1}(n)+m_{2}(n)-1}$. Finally, let $m_{3}(n):=\min \{k \geq$ $\left.0 \mid c_{m_{1}(n)+m_{2}(n)+k} \in U\right\}$. Then there exists an interval $V_{n}^{\prime \prime} \ni c_{1}$ which is mapped monotonically onto $V$ by $f^{m_{1}(n)+m_{2}(n)+m_{3}(n)-1}$, and moreover $c_{m_{1}(n)+m_{2}(n)+m_{3}(n)}$ $\in U$. Because this happens infinitely often, $r_{k}\left(c_{1}\right) \nrightarrow 0$, a contradiction.

Corollary 3.4. If $r_{n}\left(c_{1}\right) \rightarrow 0$, then $x \in B(\omega(c))$ if and only if $r_{n}(x) \rightarrow 0$.

Proof. If $x \notin B(\omega(c))$, then there exist $\delta>0$ and arbitrarily large iterates $n$ such that $d\left(f^{n}(x), \omega(c)\right)=d\left(f^{n}(x), \operatorname{orb}(c)\right)>\delta$. Since $\partial M_{n}(x) \subset \operatorname{orb}(c) \cup\{0,1\}$, it is clear that $\limsup _{n} r_{n}(x) \geq \delta$.

For the other direction, assume that $\limsup _{n} r_{n}(x)=\delta>0$. Since $r_{n} \mid \omega(c) \rightarrow$ 0 uniformly, there exists $N$ such that $r_{n} \mid \omega(c)<\frac{\delta}{4}$ for all $n \geq N$. Let $L:=$ $\sup _{J} \frac{|f(J)|}{|J|}$ (supremum taken over all subintervals of $\left[c_{2}, c_{1}\right]$ ), and $\varepsilon=\frac{\delta}{4 L^{N}}$. Assume by contradiction that $x \in B(\omega(c))$. Then there exists $M$ such that $d\left(f^{m}(x), \omega(c)\right)<$ $\varepsilon$ for all $m \geq M$.

Pick $n \geq N+M$ such that $r_{n}(x) \geq \frac{\delta}{2}$. Then there exist neighbourhoods $V \supset$ $U$ of $f^{n-N}(x)$ such that $f^{N} \mid V$ is monotone, $f^{N}(V)=\left(f^{n}(x)-\frac{\delta}{2}, f^{n}(x)+\frac{\delta}{2}\right)$, and $f^{N}(U)=\left(f^{n}(x)-\frac{\delta}{4}, f^{n}(x)+\frac{\delta}{4}\right)$. By definition of $\varepsilon$, each component of $U \backslash$ $\left\{f^{n-N}(x)\right\}$ has size $\geq \varepsilon$. As $n-N \geq M$, there exists $y \in U \cap \omega(c)$. But that means that $r_{N}(y) \geq d\left(f^{N}(y), f^{N}(\partial V)\right) \geq \frac{\delta}{4}$, contradicting the definition of $N$.

\section{The Induced Map $F$}

In this section we introduce the induced map used in sections 6 and 8 . We will construct a countable interval partition, given by points $\left\{u_{k}\right\} \cup\left\{\hat{u}_{k}\right\}$. These points are defined as follows: $\hat{u}_{1}=p$ is the orientation-reversing fixed point of $f$, and hence $u_{1}=\hat{p}$. Assume that $u_{i}$ is defined for every $i<k$.

The iterate $f^{S_{k-1}}$ maps $\left(z_{k-1}, c\right)$ and $\left(c, \hat{z}_{k-1}\right)$ diffeomorphically onto $\left(d_{k-1}, c\right)$. Let $u_{k} \in\left(z_{k-1}, z_{k}\right)$ be such that

$$
f^{S_{k-1}}\left(u_{k}\right)= \begin{cases}u_{Q(k)+1} \in\left(z_{Q(k)}, z_{Q(k)+1}\right) & \text { if } d_{k-1}<c, \\ \hat{u}_{Q(k)+1} \in\left(\hat{z}_{Q(k)+1}, \hat{z}_{Q(k)}\right) & \text { if } d_{k-1}>c .\end{cases}
$$




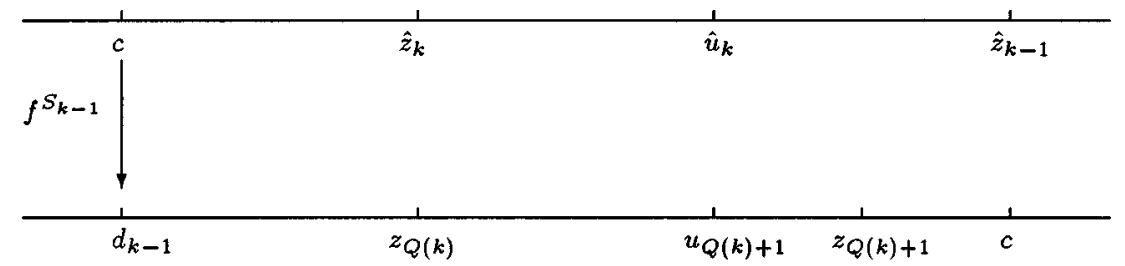

FiguRe 4.1

See Figure 4.1. Then also

$$
f^{S_{k}}\left(u_{k}\right)=f^{S_{Q(k)}} \circ f^{S_{k-1}}\left(u_{k}\right)=f^{S_{Q(k)}}\left(u_{Q(k)+1}\right)=u_{Q(Q(k)+1)+1} .
$$

Let $U=(\hat{p}, p)$ and $U_{k}=\left(u_{k-1}, u_{k}\right)$. The induced map $F$ is defined as

$$
F \mid U_{k} \cup \hat{U}_{k}=f^{S_{k-1}} .
$$

Hence

$$
F\left(U_{k}\right)=F\left(\hat{U}_{k}\right)= \begin{cases}\left(u_{Q(k)+1}, \hat{u}_{Q(Q(k-1)+1)+1}\right) & \text { if } d_{k-1}<c, \\ \left(u_{Q(Q(k-1)+1)+1}, \hat{u}_{Q(k)+1}\right) & \text { if } d_{k-1}>c .\end{cases}
$$

Clearly $F$ is a Markov map, i.e. $F$ preserves the partition $\left\{U_{k}\right\} . F$ is also an extendible Markov map. By this we mean that

$$
f^{S_{k-1}}\left(H_{S_{k-1}}\left(U_{k}\right)\right) \supset H_{S_{r-1}}\left(U_{r}\right) \text { whenever } F\left(U_{k}\right) \supset U_{r} \text {. }
$$

Here $H_{S_{k-1}}\left(U_{k}\right)$ is the maximal interval containing $U_{k}$ on which $f^{S_{k-1}}$ is monotone. Indeed, as $H_{S_{k-1}}\left(U_{k}\right)=\left(z_{k-2}, c\right)$,

$$
f^{S_{k-1}}\left(z_{k-2}, c\right)=\left(d_{Q(k-1)}, d_{k-1}\right) \supset H_{S_{r-1}}\left(U_{r}\right)=\left(z_{r-2}, c\right),
$$

for each $U_{r} \subset F\left(U_{k}\right)$. As $F$ is a Markov map, there exist well-defined cylinder sets:

$$
U_{i_{0}, \ldots i_{n-1}}=\left\{x \mid F^{m}(x) \in U_{i_{m}} \cup \hat{U}_{i_{m}} \text { for } 0 \leq m<n\right\} .
$$

Because $F$ is extendible Markov, its iterates have the same property:

Lemma 4.1. Let $V=U_{i_{0}, \ldots, i_{n-1}}$ be an arbitrary cylinder. Therefore $F^{n-1}(V)=$ $U_{k}, k=i_{n-1}$ and $F^{n}(V)=f^{r}(V)=\left(u_{Q(k)+1}, \hat{u}_{Q(Q(k-1)+1)+1}\right)$ or its involution. Then

$$
f^{r}\left(H_{r}(V)\right)=f^{S_{k-1}}\left(H_{S_{k-1}}\left(U_{k}\right)\right)=f^{S_{k-1}}\left(\left(z_{k-2}, c\right)\right)=\left(d_{Q(k-1)}, d_{k-1}\right) .
$$

Proof. Use induction.

\section{Random Walks Governed By $F$}

The main tool for the proof of the (non-)existence of absorbing Cantor sets is a random walk on the states $U_{k}$. The transitions from one state to another are given by the induced map. Write $\varphi_{n}(x)=k$ if $F^{n}(x) \in U_{k} \cup \hat{U}_{k}$. In order to prove that $x \in B(\omega(c))$, one needs to check the asymptotic behaviour of $\varphi_{n}(x)$.

Lemma 5.1. i) If $Q(k) \rightarrow \infty$ and $\lim _{n} \varphi_{n}(x)=\infty$, then $x \in B(\omega(c))$.

ii) If $r_{n}\left(c_{1}\right) \rightarrow 0$ and $x \in B(\omega(c))$, then $\lim _{n} \varphi_{n}(x)=\infty$. 
Proof. i) As $\lim _{n} \varphi(x)=\infty,\left|F^{n-1}(x)-c\right| \rightarrow 0$. By definition, $F^{n-1}(x) \in$ $U_{\varphi_{n-1}}(x)$. Because $Q(k) \rightarrow \infty$, also $\left|F^{n}(x)-d_{\varphi_{n-1}(x)-1}\right| \rightarrow 0$. It follows from the Contraction Principle that also for the intermediate iterates (i.e. for $r_{1}<r<r_{2}$ where $F^{n-1}=f^{r_{1}}$ and $\left.F^{n}=f^{r_{2}}\right), d\left(f^{r}(x), f^{r-r_{1}}(c)\right) \rightarrow 0$ as $n \rightarrow \infty$. Hence $d\left(f^{r}(x), \omega(c)\right) \rightarrow 0$.

ii) Assume by contradiction that $k_{0}:=\liminf _{n} \varphi_{n}(x)<\infty$. Let

$$
\delta:=\min _{k \leq k_{0}} \min \left\{\left|z_{k-2}-u_{k-1}\right|,\left|u_{k}-c\right|\right\} .
$$

Take $n, s$ such that $F^{n}(x)=f^{s}(x) \in U_{k}$ for some $k \leq k_{0}$. Then by Lemma 4.1, $H_{s}(x) \supset\left(z_{k-2}, c\right)$, whence $r_{s}(x) \geq \delta>0$. Since this holds for arbitrarily large $s$, $\lim \sup _{s} r_{s}(x)>0$. According to Corollary 3.4, $x \notin B(\omega(c))$.

The asymptotics of $\varphi_{n}$ can be computed from the expectation $\mathbb{E}\left(\varphi_{n}-\varphi_{n-1}\right)$, taken with respect to normalized Lebesgue measure on $U$. If $\mathbb{E}\left(\varphi_{n}-\varphi_{n-1}\right) \geq \varepsilon>0$, then we expect that $\lim _{n} \varphi_{n}(x)=\infty$ almost surely. To prove this, we will use conditional expectations. We also need boundedness of the variance. For this reason, we will use functions $\psi_{n} \leq \varphi_{n}$, which satisfy $\varphi_{n} \rightarrow \infty$ if and only if $\psi_{n} \rightarrow \infty$, but also have bounded conditional variances.

Theorem 5.2. Let $\psi_{n}: U \rightarrow \mathbb{R}, n \in \mathbb{N}$, be functions satisfying the following conditions:

- $\psi_{n-1}$ is constant on each cylinder $U_{i_{0}, \ldots i_{n-1}}$.

- There exist $k_{1} \in \mathbb{N}$ and $\delta>0$ such that for every cylinder $U_{i_{0}, \ldots i_{n-1}}$ with $i_{n-1} \geq k_{1}, \mathbb{E}\left(\psi_{n}-\psi_{n-1} \mid U_{i_{0}, \ldots i_{n-1}}\right) \geq \delta$.

- $\operatorname{Var}\left(\bar{\psi}_{n}-\psi_{n-1} \mid U_{i_{0}, \ldots i_{n-1}}\right)$ is uniformly bounded.

Then the set $X=\left\{x \in U \mid \psi_{n}(x) \rightarrow \infty\right\}$ has positive Lebesgue measure.

Proof. We first restrict ourselves to $X_{0}=\left\{x \mid \varphi_{n}(x) \geq k_{1}\right.$ for all $\left.n\right\}$. Define $\Psi_{m}=\left(\psi_{m}-\psi_{m-1}\right)-\mathbb{E}\left(\left(\psi_{m}-\psi_{m-1}\right) \mid U_{i_{0} \ldots i_{m-1}}\right)$. Then $\mathbb{E}\left(\Psi_{m} \mid U_{i_{0} \ldots i_{m-1}}\right)=0$ and there exists $V$ such that $\operatorname{Var}\left(\Psi_{m} \mid U_{i_{0} \ldots i_{m-1}}\right)=\mathbb{E}\left(\Psi_{m}^{2} \mid U_{i_{0} \ldots i_{m-1}}\right)<V$ for all $m$ and all cylinders $U_{i_{0} \ldots i_{m-1}}$. Let $T_{n}:=\sum_{m=1}^{n} \Psi_{m}$, so $\mathbb{E}\left(T_{1}^{2}\right)=\mathbb{E}\left(\Psi_{1}^{2}\right) \leq V$. By assumption $T_{n-1}$ is constant on each set $U_{i_{0} \ldots i_{n-1}}$. Suppose by induction that $\mathbb{E}\left(T_{n-1}^{2}\right) \leq(n-1) V ;$ then

$$
\begin{aligned}
\mathbb{E}\left(T_{n}^{2}\right) & =\mathbb{E}\left(T_{n-1}^{2}\right)+\mathbb{E}\left(\Psi_{n}^{2}\right)+2 \mathbb{E}\left(\Psi_{n} T_{n-1}\right) \\
& \leq(n-1) V+V+2 \sum_{U_{i_{0} \ldots i_{n-1}}} \mathbb{E}\left(\Psi_{n} T_{n-1} \mid U_{i_{0} \ldots i_{n-1}}\right) \\
& \leq n V+2 \sum_{U_{i_{0} \ldots i_{n-1}}} T_{n-1} \cdot \mathbb{E}\left(\Psi_{n} \mid U_{i_{0} \ldots i_{n-1}}\right)=n V .
\end{aligned}
$$

By the Chebyshev inequality, $P\left(\left|T_{n}\right|>n \varepsilon\right) \leq \frac{n V}{n^{2} \varepsilon^{2}}=\frac{V}{n \varepsilon^{2}}$. In particular, $P\left(\left|T_{n^{2}}\right|>n^{2} \varepsilon\right) \leq \frac{V}{n^{2} \varepsilon^{2}}$. Therefore $\sum_{n} P\left(\left|T_{n^{2}}\right|>n^{2} \varepsilon\right)<\infty$ and, by the BorelCantelli Lemma,

$$
P\left(\left|T_{n^{2}}\right|>n^{2} \varepsilon \text { infinitely often }\right)=0 .
$$

As $\varepsilon$ is arbitrary, $T_{n^{2}} / n^{2} \rightarrow 0$ a.s. Now for the intermediate values of $n$, let

$$
D_{n}:=\max _{n^{2}<k<(n+1)^{2}}\left|T_{k}-T_{n^{2}}\right| .
$$


Because $\left|T_{k}-T_{n^{2}}\right|=\left|\sum_{j=n^{2}+1}^{k} \Psi_{j}\right|, \mathbb{E}\left(\left|T_{k}-T_{n^{2}}\right|^{2}\right) \leq\left(k-n^{2}\right) V \leq 2 n V$. Hence

$$
\mathbb{E}\left(D_{n}^{2}\right) \leq \mathbb{E}\left(\sum_{k=n^{2}+1}^{(n+1)^{2}-1}\left|T_{k}-T_{n^{2}}\right|^{2}\right) \leq \sum_{k=n^{2}+1}^{(n+1)^{2}-1} 2 n V=4 n^{2} V .
$$

Using Chebyshev's inequality again, we obtain

$$
P\left(D_{n} \geq n^{2} \varepsilon\right) \leq \frac{4 n^{2} V}{n^{4} \varepsilon^{2}}=\frac{4 V}{n^{2} \varepsilon^{2}} .
$$

Hence $P\left(D_{n} \geq n^{2} \varepsilon\right.$ infinitely often $)=0$, and $D_{n} / n^{2} \rightarrow 0$ a.s. Combining things and taking $n^{2} \leq k<(n+1)^{2}$, we get

$$
\frac{\left|T_{k}\right|}{k} \leq \frac{\left|T_{n^{2}}\right|+D_{n}}{n^{2}} \rightarrow 0 \text { a.s. }
$$

Because $\psi_{m}-\psi_{m-1}=\Psi_{m}+\mathbb{E}\left(\psi_{m}-\psi_{m-1} \mid U_{i_{0} \ldots i_{m-1}}\right) \geq \Psi_{m}+\delta$,

$$
\liminf _{n \rightarrow \infty} \frac{1}{n} \sum_{i=2}^{n}\left(\psi_{i}-\psi_{i-1}\right) \geq \liminf _{n \rightarrow \infty} \frac{1}{n} T_{n}+\delta \geq \delta \text { a.s. }
$$

Hence $\psi_{n}(x) \rightarrow \infty$ for a.e. $x \in X_{0}$. Therefore $\frac{\left|X_{0} \cap X \cap U_{k}\right|}{\left|U_{k}\right|} \rightarrow 1$ as $k \rightarrow \infty$, whence $|X|>0$.

\section{Maps Having Absorbing Cantor Sets}

Theorem 6.1. Let $f$ be a non-renormalizable S-unimodal map with critical order $\ell<\infty$. Let its kneading map $Q$ satisfy the following properties: There exists $N, k_{1} \in \mathbb{N}$ such that for all $k \geq k_{1}$,

$$
Q(k+1)>Q^{2}(k)+1
$$

and

$$
Q(k) \geq k-N .
$$

Then there exists $\ell_{0}=\ell_{0}(N)$ such that, if $\ell \geq \ell_{0}$, then $f$ has an absorbing Cantor set.

Remark. Condition (6.1) is rather unnatural. It will be used effectively in Lemma 10.2. Note that (6.1) is satisfied if $f$ is finitely renormalizable (use Lemma 2.3) and $Q$ is eventually non-decreasing. Note also that (6.1) immediately implies (2.4). Hence Lemma 2.5 applies. We need (6.1) for the estimates, but we don't believe that it really affects the existence of an absorbing Cantor set.

According to Theorem 5.2 it suffices to check certain conditional expectations and variances. This will be done in Proposition 6.2. This proposition uses the following metrical conditions: There exist $C, \ell_{0} \geq 1$ and $k_{1} \in \mathbb{N}$ such that for all $\ell \geq \ell_{0}$ and $k \geq k_{1}$

$$
\frac{1}{C \ell} \leq \frac{\left|u_{k-1}-u_{k}\right|}{\left|u_{k-1}-c\right|} \leq \frac{C}{\ell}
$$

and

$$
\frac{1}{C \ell} \leq \frac{\left|d_{k-1}-u_{Q(k)+1}\right|}{\left|u_{Q(k)+1}-c\right|} \leq \frac{C}{\ell} .
$$

The proofs of these statements are a generalization of the proofs in [BKNS]. They are very technical; we put them in section 10 . 


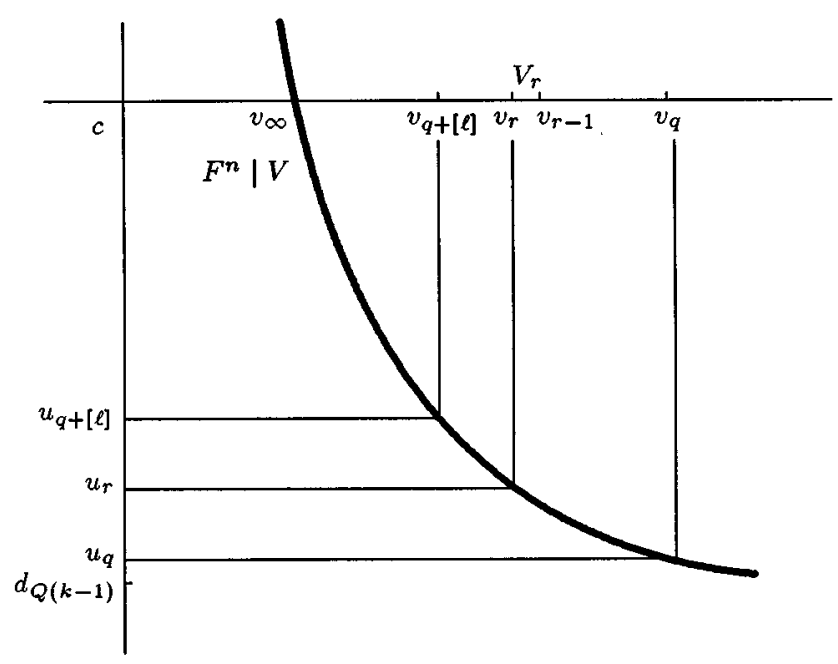

Figure 6.1

Proposition 6.2. Let $f$ be a Fibonacci-like S-unimodal map having critical order $\ell$ and kneading map $Q$. Let $k_{1}$ be such that (6.3) and (6.4) hold. Then there exists $\ell_{0}<\infty$ such that if $\ell \geq \ell_{0}$, and $U_{\varphi_{0}, \ldots \varphi_{n-1}}$ is a cylinder with $\varphi_{n-1}>k_{1}$, then $\mathbb{E}\left(\varphi_{n}-\varphi_{n-1} \mid U_{\varphi_{0}, \ldots \varphi_{n-1}}\right)>1$ and $\operatorname{Var}\left(\varphi_{n}-\varphi_{n-1} \mid U_{\varphi_{0}, \ldots \varphi_{n-1}}\right)$ is uniformly bounded.

Proof. Let $V=U_{\varphi_{0}, \ldots \varphi_{n-1}}$ be any cylinder set such that $\varphi_{n-1} \geq k_{1}$. Since $F$ is extendible Markov, the Koebe space corresponding to $F^{n} \mid V$ depends only on the last application of $F$ in the composition. In other words, suppose that $F^{n}=f^{s}$ and $F^{n-1}(V)=U_{k}$; then $f^{s}\left(H_{s}(V)\right)=f^{S_{k-1}}\left(\left(c, z_{k-2}\right)\right)=\left(d_{Q(k-1)}, d_{k-1}\right)$.

To be definite, assume that $d_{Q(k-1)}<c<d_{k-1}$. We will only calculate the conditional expectation and variance on the part $V^{0} \subset V$ that is mapped to $\left(u_{Q(Q(k-1)+1)+1}, c\right)$. The estimates for the part $V^{1}$ of $V$ which is mapped onto $\left(c, \hat{u}_{Q(k)+1}\right)$ are similar.

Abbreviate $q=Q(Q(k-1)+1)+1>k-2 N$. For $r \geq q$, let $v_{r} \in V$ be such that $F^{n}\left(v_{r}\right)=u_{r}$ and $F^{n}\left(v_{\infty}\right)=c$. Also let $V_{r}=\left(v_{r}, v_{r-1}\right)$ (see Figure 6.1).

By $(6.3),\left(1-\frac{C}{\ell}\right) \leq \frac{\left|u_{i+1}-c\right|}{\left|u_{i}-c\right|} \leq\left(1-\frac{1}{C \ell}\right)$, whence

$$
\left(1-\frac{C}{\ell}\right)^{i-j} \leq \frac{\left|u_{i}-c\right|}{\left|u_{j}-c\right|} \leq\left(1-\frac{1}{C \ell}\right)^{i-j} .
$$

Using also (6.4), we obtain that for $\ell$ sufficiently large, and $k<r \leq q+\ell$, there exist $K=K(C, \ell)>0$ such that

$$
\begin{aligned}
\frac{\left|d_{Q(k-1)}-u_{q}\right|}{\left|d_{Q(k-1)}-u_{r}\right|} & \geq \frac{\left|d_{Q(k-1)}-u_{q}\right|}{\left|u_{q}-c\right|} \frac{\left|u_{q}-c\right|}{\left|u_{q-1}-c\right|} \frac{1}{1-\frac{\left|u_{r}-c\right|}{\left|u_{q-1}-c\right|}} \\
& \geq \frac{1}{C \ell} \frac{1-\frac{C}{\ell}}{1-\left(1-\frac{C}{\ell}\right)^{r-q}} \geq \frac{K}{r-q},
\end{aligned}
$$


and

$$
\begin{aligned}
\frac{\left|U_{r}\right|}{\left|u_{q}-u_{r-1}\right|} & =\frac{\left|U_{r}\right|}{\left|u_{r-1}-c\right|} \frac{\left|u_{r-1}-c\right|}{\left|u_{q}-c\right|} \frac{1}{1-\frac{\left|u_{r-1}-c\right|}{\left|u_{q}-c\right|}} \\
& \geq \frac{1}{C \ell} \frac{\left(1-\frac{C}{\ell}\right)^{r-1-q}}{1-\left(1-\frac{C}{\ell}\right)^{r-1-q}} \geq \frac{K}{r-q} .
\end{aligned}
$$

These relations and the expansion of cross-ratios (with $L=U_{r}, J=\left(u_{r-1}, u_{q}\right.$ ) and $\left.R=\left(u_{q}, d_{Q(k-1)}\right)\right)$ yield, for $k<r \leq q+\ell$,

$$
\frac{\left|V_{r}\right|}{\left|v_{r-1}-v_{q}\right|}=\frac{|l|}{|j|} \geq \frac{|R|}{|T|} \frac{|L|}{|J|} \geq \frac{\left|d_{Q(k-1)}-u_{q}\right|}{\left|d_{Q(k-1)}-u_{r}\right|} \frac{\left|U_{r}\right|}{\left|u_{q}-u_{r-1}\right|} \geq \frac{K^{2}}{(r-q)^{2}} .
$$

Hence the conditional expectation

$$
\begin{gathered}
\mathbb{E}\left(\varphi_{n}-k \mid V^{0}\right)=\frac{1}{\left|V^{0}\right|} \sum_{j}(j-k)\left|V_{j}\right| \\
\geq \frac{1}{\left|V^{0}\right|}\left\{-2 N\left|v_{k}-v_{q}\right|+(\ell-2 N)\left|v_{q+[\ell]}-v_{\infty}\right|\right. \\
\left.\quad+\sum_{j=k+1}^{q+[\ell]} K^{2} \frac{j-k}{(j-q)^{2}}\left|v_{j-1}-v_{q}\right|\right\} \\
\geq \frac{1}{\left|V^{0}\right|}\left\{\left(\frac{K^{2}}{2} \log \ell-2 N\right)\left|v_{k}-v_{q}\right|+(\ell-2 N)\left|v_{q+[\ell]}-v_{\infty}\right|\right\} .
\end{gathered}
$$

for $\ell$ sufficiently large. Hence $\mathbb{E}\left(\varphi_{n}-k \mid V^{0}\right) \rightarrow \infty$ as $\ell \rightarrow \infty$.

Let us compute the variance. For $j \geq k$, the interval $\left(u_{j}, c\right)$ has Koebe space $\left(d_{Q(k-1)}, u_{j}\right)$ which is at least of order $\frac{1}{\ell}$. This gives a distortion bound of $\mathcal{O}\left(\ell^{2}\right)$ :

$$
\begin{aligned}
\operatorname{Var}\left(\varphi_{n}-k \mid V^{0}\right) & \leq \mathbb{E}\left(\left(\varphi_{n}-k\right)^{2} \mid V^{0}\right)=\frac{1}{\left|V^{0}\right|} \sum_{j}(j-k)^{2}\left|V_{j}\right| \\
& \leq 4 N^{2}+\sum_{j>k}(j-k)^{2} \frac{\left|V_{j}\right|}{\left|V^{0}\right|} \\
& \leq 4 N^{2}+\mathcal{O}\left(\ell^{2}\right) \sum_{j>k}(j-k)^{2} \frac{\left|u_{j}-c\right|}{\left|u_{k}-c\right|} \\
& \leq 4 N^{2}+\mathcal{O}\left(\ell^{2}\right) \sum_{j>k}(j-k)^{2}\left(1-\frac{1}{C \ell}\right)^{j-k}=4 N^{2}+\mathcal{O}\left(\ell^{5}\right) .
\end{aligned}
$$

This concludes the proof.

Proof of Theorem 6.1. Combine Proposition 6.2, Theorem 5.2 and Lemma 5.1.

\section{Estimates ON $\frac{\left|u_{k}-u_{k+1}\right|}{\left|u_{k}-c\right|}$}

In this section we concentrate on the relative space between $u_{k}$ and $u_{l}$ (or similarly, the relative space between $z_{k}$ and $z_{l}$ ) for $l>k$. Let $\varepsilon>0$ be arbitrary. We introduce the function $\rho_{\varepsilon}$ to measure how much $\left|z_{l}-c\right|$ is smaller than $\left|z_{k}-c\right|$ in factors $1-\varepsilon:\left|z_{l}-c\right| \approx(1-\varepsilon)^{\rho_{\varepsilon}(l)-\rho_{\varepsilon}(k)}\left|z_{k}-c\right|$. More precisely, we define 
$\rho=\rho_{\varepsilon}: \mathbb{N} \rightarrow \mathbb{N}$ by $\rho(0):=0$, and if $l \leq k-1$ is the largest integer such that $\rho(l)=\rho(k-1)$, then

$$
\rho(k):= \begin{cases}\rho(k-1)+1 & \text { if } \frac{\left|z_{l}-z_{k}\right|}{\left|z_{l}-c\right|} \geq \varepsilon \\ \rho(k-1) & \text { otherwise. }\end{cases}
$$

Hence $\rho(k-1) \leq \rho(k) \leq k$ for each $k \geq 1$, and for $k \geq l, \frac{\left|z_{k}-c\right|}{\left|z_{l}-c\right|} \leq(1-\varepsilon)^{\rho(k)-\rho(l)}$. Because $z_{k} \in\left(u_{k}, u_{k-1}\right)$ for all $k$, also $\frac{\left|u_{k+1}-c\right|}{\left|u_{l}-c\right|} \leq(1-\varepsilon)^{\rho(k)-\rho(l)}$.

In this section we will try to estimate $\rho$ using conditions on $Q$. For instance, if $Q$ is eventually non-decreasing, then $\rho(k)=\rho(k)+1$ for $k$ sufficiently large and $\varepsilon$ sufficiently small. (One should think of $\varepsilon=\mathcal{O}\left(\ell^{-2}\right)$.) Hence $\left|u_{k}-c\right|$ decrease exponentially in this case.

Remark. One of the main difficulties in getting estimates for $\frac{\left|z_{k}-c\right|}{\left|z_{k-1}-c\right|}$ for arbitrary unimodal maps is the occurrence of almost saddle node bifurcations. We speak of an almost saddle node bifurcation of period $n$ if the graph of the central branch of $f^{n}$ is disjoint from, but almost tangent to, the diagonal. At the bifurcation itself, i.e. when the graph of the central branch is tangent, a neutrally attracting $n$-periodic point, say $q$, is created. Without loss of generality, assume that $q<c$. Then the precritical points $z_{k}$ accumulate on $q$. By a continuity argument one can show that close before the bifurcation, the precritical points $z_{k}, z_{k+1}, \ldots, z_{k+j_{0}}$ cluster together around the spot 'where $q$ is going to appear'. Here the period $n=S_{k}-S_{Q^{2}(k)}$ and $j_{0}$ can be arbitrarily large. Therefore $\rho_{\varepsilon}(i)$ can be constant for arbitrarily long pieces. However, we think that the occurrence of almost saddle node bifurcations does not affect the (non-)existence of absorbing Cantor sets. (The existence of absolutely continuous invariant probability measures is a totally different matter; cf. [B1].) As we cannot support this remark by rigid estimates, we will exclude almost saddle node bifurcations by assumption.

The estimates in this section rely on one technique developed by Martens [Ma1][Ma2], and involve nice points. We will use the following weaker version of niceness:

$$
f^{j}(x) \notin(x, \hat{x}) \text { for every } j<S_{k},
$$

where $S_{k}$ is minimal such that $c \in f^{S_{k}}((x, c))$.

Proposition 7.1. There exists $\delta=\delta(\ell)>0$ with the following property: Let $x \in$ $\left(z_{k-1}, z_{k}\right)$ satisfy (7.1). If the interval $J$ and $n \in \mathbb{N}$ are such that $f^{n} \mid J$ is the monotone first visit of $J$ to $(x, \hat{x})$, then there exists $T \supset J$ such that $f^{n} \mid T$ is monotone and $f^{n}(T)$ contains a $\delta$-scaled neighbourhood of $(x, \hat{x})$.

Proof. Let $M:=\left(x^{f}, c_{1}\right)$. Then $f^{S_{k}-1}(M) \ni c$, and by assumption $f^{j}(M) \cap(x, \hat{x})=$ $\emptyset$ for $j<S_{k}-1$. Let $m<S_{k}$ be such that $\left|f^{m}(M)\right| \leq\left|f^{j}(M)\right|$ for all $j<S_{k}$. Let $r, l<S_{k}$ be such that $f^{r}(M)$ and $f^{l}(M)$ are the closest right and left neighbours of $f^{m}(M)$ that are still disjoint from $f^{m}(M)$. Both neighbours need not exist, for instance if $m \in\{0,1\}$. In those cases define the disjoint right or left neighbour as the appropriate boundary point of $I$. It follows that $\left[f^{l}(M), f^{r}(M)\right]$ contains a 1-scaled neighbourhood of $f^{m}(M)$. (This holds true also if the closest disjoint left or right neighbour does not properly exist and $|x-c|$ is sufficiently small.)

Let $H$ be the maximal interval such that $f^{m} \mid H$ is monotone and $f^{m}(H) \subset$ $\left[f^{l}(M), f^{r}(M)\right]$. We claim that

$$
f^{m}(H)=\left[f^{l}(M), f^{r}(M)\right] .
$$


Suppose by contradiction that $f^{m}(H) \subsetneq\left[f^{l}(M), f^{r}(M)\right]$. Let $L$ be a component of $H \backslash M$. If $\partial I \cap \partial L \neq \emptyset$, then also $f^{m}(\partial L) \cap \partial I \neq \emptyset$, and we already have a contradiction. We can assume, by maximality of $H$, that there exist $j<m$ such that $c \in f^{j}(\partial L)$. As $f^{j}(M) \cap(x, \hat{x})=\emptyset$, it follows that $f^{m}(L) \supset f^{m-j-1}(M)$ and $f^{m-j-1}(M) \cap f^{m}(M)=\emptyset$. This contradicts the definition of closest disjoint neighbour, proving the claim.

Due to the expansion of cross-ratio, we can pull $\left[f^{l}(M), f^{r}(M)\right]$ back along the orbit $M, f(M), \ldots ., f^{m}(M)$ and show that $H$ contains a $\frac{1}{3}$-scaled neighbourhood of $M$. Let $H^{\prime}=f^{-1}(H)$; then $H^{\prime}$ contains a $\delta$-scaled neighbourhood of $(x, \hat{x})$, where $\delta=\delta(\ell)=\mathcal{O}\left(\frac{1}{\ell}\right)$.

Now let $J$ and $n$ be as in the statement of the proposition. To finish the proof, it suffices to show that there exists a neighbourhood $T \supset J$ such that $f^{n}$ maps $T$ monotonically onto $H^{\prime}$. Let $T \supset J$ be the maximal neighbourhood of $J$ such that $f^{n} \mid T$ is monotone and $f^{n}(T) \subset H^{\prime}$. Suppose by contradiction that $f^{n}(T) \subsetneq H^{\prime}$. Let $L$ be a component of $T \backslash J$. By maximality, there exists $j<n$ such that $c \in \partial f^{j}(L)$. By assumption $f^{j}(J) \cap(x, \hat{x})=\emptyset$, so $f^{n}(L) \supset f^{n-j-1}(M)$ and $f^{n-j-1}(M)$ is disjoint from $(x, \hat{x})$. As $f^{m+1}$ is monotone on each component of $H^{\prime} \backslash\{c\}$, also $f^{n+m+1} \mid L$ is monotone, and $f^{n+m+1}(L) \supset f^{n+m-j}(M)$, which is disjoint from $f^{m}(M)$. However $f^{S_{k}}((x, \hat{x})) \ni c$, so $n+m-j<S_{k}$. Again, this contradicts the definition of the closest disjoint neighbours of $f^{m}(M)$.

Remark. In the second half of the proof it is not necessary that $f^{j}(J)$ is disjoint from $(x, \hat{x})$ for all $j<n$. It suffices if $f^{j}(J) \cap(x, \hat{x})=\emptyset$ when $c \in \partial f^{j}(L)$.

Corollary 7.2. There exist $K(\ell)$ and $\varepsilon(\ell)>0$ such that if $c_{j} \notin\left(d_{k}, \hat{d}_{k}\right)$ for all $0<j<S_{k+1}$, then $\operatorname{dis}\left(f^{S_{k}-1},\left(z_{k}^{f}, c^{f}\right)\right) \leq K(\ell)$ and $\frac{\left|z_{k-1}-z_{k}\right|}{\left|z_{k-1}-c\right|} \geq \varepsilon(\ell)$.

In particular, $\rho_{\varepsilon}(k)=\rho_{\varepsilon}(k-1)+1$. If $Q$ is (eventually) non-decreasing, then $d_{k}$ is a closest return for every $k$ sufficiently large (Lemma 2.4). This implies that there exist $k_{0}, R \in \mathbb{N}$ such that $\rho(k)=k-R$ for all $k \geq k_{0}$.

Proof. We use Proposition 7.1 with $x=d_{k}$. Because $c_{j} \notin\left(d_{k}, \hat{d}_{k}\right)$ for $0<j<S_{k+1}$, we have $f^{j}\left(\left(d_{k}, \hat{d}_{k}\right)\right) \cap\left(d_{k}, \hat{d}_{k}\right)=\emptyset$ for $0<j<S_{Q(k+1)}$.

Let $b$ be the middle point between $c$ and $\hat{d}_{k}$, and let $J,\left(z_{k}^{f}, c^{f}\right) \subset J \subset\left(z_{k-1}^{f}, c^{f}\right)$, be such that $f^{S_{k}-1}(J)=\left(b, d_{k}\right)$. Let $T=H_{S_{k}-1}\left(c^{f}\right)$; then $z_{k-1}^{f} \in \partial T$. As $f^{S_{k}-1}\left(z_{k-1}^{f}\right)=d_{Q(k)} \notin\left(d_{k}, \hat{d}_{k}\right), f^{S_{k}-1}(T)$ contains a one-sided $\frac{1}{4}$-scaled neighbourhood of $\left(b, d_{k}\right)$ at the side of $b$.

Because $c_{j} \notin\left(d_{k}, \hat{d}_{k}\right)$ for $j<S_{k}$, one can show that $f^{S_{k}-1}(T)$ also contains the component of $H^{\prime} \backslash\{c\}$ containing $d_{k}$. Here $H^{\prime}$ is as in the proof of Proposition 7.1. Hence $f^{S_{k}-1}(T)$ contains the necessary $\delta$-scaled neighbourhood of $\left(b, d_{k}\right)$, where $\delta=\delta(\ell)$ is as in Proposition 7.1.

Hence $f^{S_{k}-1} \mid J$ and $f^{S_{k}-1} \mid\left(z_{k}^{f}, c^{f}\right)$ have bounded distortion. Moreover, $|J| \geq$ $(1+\mathcal{O}(\delta))\left|z_{k}^{f}-c^{f}\right|$, so the second statement follows by non-flatness.

The next corollary gives a weaker condition implying $\rho(k)=\rho(k-1)+1$. Let $p_{k} \in\left(z_{k-1}, z_{k}\right) \cup\left(\hat{z}_{k}, \hat{z}_{k-1}\right)$ be the orientation-reversing $S_{k-1}$-periodic point of $f$. By definition of closest precritical point, $f^{j}\left(\left(z_{k-1}, z_{k}\right)\right) \cap\left(z_{k-1}, \hat{z}_{k-1}\right)=\emptyset$ for all $0<j<S_{k-1}$. Hence $p_{k}$ is a nice point. 


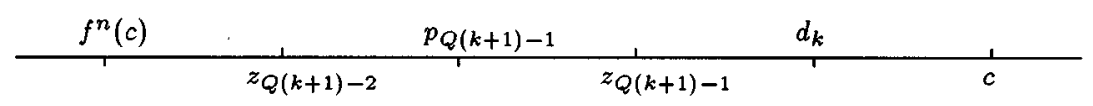

FiguRe 7.1

Corollary 7.3. There exist $\delta(\ell), \varepsilon(\ell)>0$ with the following properties: For $k$ arbitrary, let $n$ be such that $f^{n-1}\left(t_{k}^{f}\right)=c$. Assume that $f^{n}(c) \notin\left(p_{Q(k+1)-1}, \hat{p}_{Q(k+1)-1}\right)$. Then $f^{S_{k}-1} \mid\left(z_{k}^{f}, c^{f}\right)$ has Koebe space $\delta$ on the side of $d_{k}$. If also $\left|d_{k}-c\right|<\left|d_{Q(k)}-c\right|$, then $\frac{\left|z_{k-1}-z_{k}\right|}{\left|z_{k-1}-c\right|} \geq \varepsilon$.

The assumptions are such that the positions in Figure 7.1 hold. For example, if (2.4) holds, then Lemma 2.5 shows that $n=S_{k}-S_{Q^{2}(k)}$ for $k$ sufficiently large. One can verify that the assumptions of Corollary 7.3 hold if $Q(k+1)>Q^{2}(k)+2$ and $Q(k+1)>Q(Q(k)+1)$ for $k$ sufficiently large.

Proof. The proof is similar to the proof of Corollary 7.2. Take $x=p_{Q(k+1)-1}$. Therefore $x$ is nice and $d_{k} \in(x, \hat{x})$. Let $J \ni c^{f}$ be the maximal neighbourhood of $c^{f}$ such that $f^{S_{k}-1}(J) \subset(x, \hat{x})$. Let $L \subset\left(c^{f}, 1\right)$ be the maximal interval adjacent to $J$ on which $f^{S_{k}-1}$ is monotone. According to the remark below the proof of Proposition 7.1, it suffices to check that $f^{j}(J) \cap(x, \hat{x})=\emptyset$ if $\partial f^{j}(L) \ni c$. By assumption $j=n-1$, and $f^{n}(c) \notin(x, \hat{x})$. Hence $f^{n-1}(J) \cap(x, \hat{x})=\emptyset$. Therefore the (one-sided) Koebe space is sufficiently large. If also $\left|d_{Q(k)}-c\right|>\left|d_{k}-c\right|$, then $\frac{\left|z_{k-1}-z_{k}\right|}{\left|z_{k-1}-c\right|} \geq \varepsilon$ follows by non-flatness.

\section{Maps Having No Absorbing Cantor Sets}

Theorem 8.1. Let $Q$ be a kneading map satisfying

$$
k-Q(k) \rightarrow \infty \text { as } k \rightarrow \infty .
$$

Let $f$ be a non-flat S-unimodal map and have kneading map $Q$. Assume also that there exist $N \in \mathbb{N}$ and $\varepsilon>0$ such that

$$
\rho_{\varepsilon}(k+N)>\rho_{\varepsilon}(k),
$$

for all $k$ sufficiently large. Then $f$ has no absorbing Cantor set.

From the previous section one can derive topological conditions that imply (8.2). The simplest such condition is that $Q$ is (eventually) non-decreasing. Hence Theorem 8.1 yields that every non-flat S-unimodal map with $k-Q(k) \rightarrow \infty$ and $Q(k+1) \geq Q(k)$ for $k$ sufficiently large has no absorbing Cantor set.

Instead of $F$ we will used a reinduced map $G$, which has better distortion properties. In this section let $\varphi_{n}^{G}(x)=k$ if $G^{n}(x)=k$. Clearly $\varphi_{n}^{G}(x) \rightarrow \infty$ if $\varphi_{n}(x) \rightarrow \infty$. For the proof of Theorem 8.1 we use Theorem 5.2. Therefore we need to check certain conditional expectations and variances. But even the adjusted functions $\varphi_{n}^{G}$ are not adequate. First of all, $\varphi_{n}^{G}$ are, so to speak, in the wrong direction: We need to prove that $\varphi_{n}^{G}(x)$ does not tend to infinity for most points. Second, $\varphi_{n}^{G}$ will not give us bounded variances. Therefore we will use a certain truncation of $-\varphi_{n}^{G}$. 
Proof of Theorem 8.1. Let $k_{0}$ be such that $\rho_{\varepsilon}(k+N)>\rho_{\varepsilon}(k)$ for all $k \geq k_{0}$. Let $K:=\left(\frac{1+\varepsilon}{\varepsilon}\right)^{2}$ be a distortion bound, and let $M \in \mathbb{N}$ be so large that

$$
M \varepsilon-K \sum_{j \geq 0} j(1-\varepsilon)^{j / N} \geq 1
$$

Let $k_{1} \geq k_{0}+M+N+2$ be so large that $Q(k) \leq k-M-N-2$ for all $k \geq k_{1}$.

Now we define the reinduced map $G$. Let $x$ be such that $F^{n}(x)$ is defined for all $x$. Let $i=i(x) \geq 1$ be the smallest integer which satisfies one of the following properties. (Take $r$ such that $F^{i-1}(x) \in U_{r} \cup \hat{U}_{r}$.)

- $r<k_{1}$.

- $r \geq k_{1}$ and there exists a neighbourhood $V \ni x$ such that $F^{i} \mid V$ is monotone and $F^{i}(V) \supset\left(u_{k_{0}}, \hat{u}_{k_{0}}\right)$.

- $r \geq k_{1}$ and $F^{i}(x) \in\left(u_{r-M-N-1}, \hat{u}_{r-M-N-1}\right)$.

Then

$$
G(x):=F^{i}(x) .
$$

For $s \geq k_{1}, G \mid U_{s}$ consists of a middle branch $G: V \rightarrow\left(u_{s-M-N-1}, \hat{u}_{s-M-N-1}\right)$ coinciding with $F: V \rightarrow\left(u_{s-M-N-1}, \hat{u}_{s-M-N-1}\right)$. All other branches of $G \mid U_{k}$ are longer. Note that $G$ is well-defined for a.e. $x \in U$ : Either $x \in \bigcup_{k, j} f^{-j}\left(u_{k} \cup \hat{u}_{k}\right)$ (which applies to countably many points only), or $i(x)$ is finite. Indeed, if $i(x)=\infty$, then $\varphi_{n}(x)<\varphi_{n-1}(x)$ for all $n$. This is of course impossible.

The branches of $G^{n}$ have nice distortion properties. First of all, as $G$ is an induced map of an extendible Markov map $F, G$ is also extendible Markov, and it inherits the Koebe spaces of $F$. Before giving detailed distortion results, we introduce some more notation. Suppose $V \subset U_{\varphi_{0}^{G}, \ldots . \varphi_{n-1}^{G}}$ is a branch-domain of $G^{n}$. Let $V_{r}^{+} \subset V$ be such that $G^{n}\left(V_{n}^{+}\right)=\hat{U}_{r}$, and let $V_{r}^{-} \subset V$ be such that $G^{n}\left(V_{n}^{-}\right)=U_{r}$. Assume $\varphi_{n-1}^{G}(V)=s \geq k_{1}$. Hence $G^{n}(V)$ contains an $\varepsilon$-scaled neighbourhood of $\left(u_{s}, \hat{u}_{s}\right)$. In particular, $G^{n}(V)$ contains an $\varepsilon$-scaled neighbourhood of $\bigcup_{r \geq s} G^{n}\left(V_{r}^{+} \cup V_{r}^{-}\right)$. By the Koebe Principle, it follows that

$$
K \frac{\left|G^{n}(V)\right|}{|V|} \geq \frac{\left|\bigcup_{r \geq s} G^{n}\left(V_{r}^{+} \cup V_{r}^{-}\right)\right|}{\left|\bigcup_{r \geq s}\left(V_{r}^{+} \cup V_{r}^{-}\right)\right|} \geq \frac{1}{K} \frac{\left|G^{n}(V)\right|}{|V|} .
$$

Assume that $G^{n}(V)=\left(u_{a}, \hat{u}_{b}\right)$, and let $W \subset V$ be the interval such that $G^{n}(W)=$ $\left(u_{a}, u_{s-M}\right)$ or $\left(\hat{u}_{s-M}, \hat{u}_{b}\right)$. Then we can estimate $\frac{|W|}{|V|}$ from below. Indeed, take $x \in V$ such that $G^{n}(x)=c$, and let $W_{0} \supset W$ and $W_{1}$ be the components of $V \backslash\{x\}$. Let $h: I \rightarrow V$ be the (unique) surjective Möbius transformation such that $\frac{|W|}{|V|}=\frac{\left|h^{-1}(W)\right|}{\left|h^{-1}(V)\right|}$ and $\frac{\left|h^{-1}\left(W_{0} \backslash W\right)\right|}{\left|h^{-1}\left(W_{1}\right)\right|}=1$. Then the situation is as in Figure 8.1.

As $G^{n}(V) \supset\left(u_{s-M}, \hat{u}_{s-M}\right),\left|G^{n}\left(W_{1}\right)\right| \geq\left|G^{n}\left(W_{0} \backslash W\right)\right|$. By the expansion of cross-ratio

$$
\frac{|W|}{|V|}=\frac{|l|}{|t|} \geq \frac{|j|}{|r|} \frac{|L|}{|T|} \frac{|R|}{|J|}=1 \cdot \frac{\left|G^{n}(W)\right|}{\left|G^{n}(V)\right|} \frac{\left|G^{n}\left(W_{1}\right)\right|}{\left|G^{n}\left(W_{0} \backslash W\right)\right|} \geq \frac{\left|G^{n}(W)\right|}{\left|G^{n}(V)\right|} .
$$

Define $\psi_{0}(x)=-\varphi_{0}^{G}(x)=-\varphi_{0}(x)$ and

$$
\psi_{n}(x)=\psi_{n-1}-\max \left\{\varphi_{n}^{G}(x)-\varphi_{n-1}^{G}(x),-M\right\} .
$$

Clearly $-\psi_{n}(x) \geq \varphi_{n}^{G}(x)$ for every $n$. We claim that for all cylinders $U_{\varphi_{0}^{G}, \ldots . \varphi_{n-1}^{G}}$ with $\varphi_{n-1}^{G} \geq k_{1}, \mathbb{E}\left(\psi_{n}-\psi_{n-1} \mid U_{\varphi_{0}^{G}, \ldots \varphi_{n-1}^{G}}\right) \geq 1$ and $\operatorname{Var}\left(\psi_{n}-\psi_{n-1} \mid U_{\varphi_{0}^{G}, \ldots \varphi_{n-1}^{G}}\right)$ is uniformly bounded. 


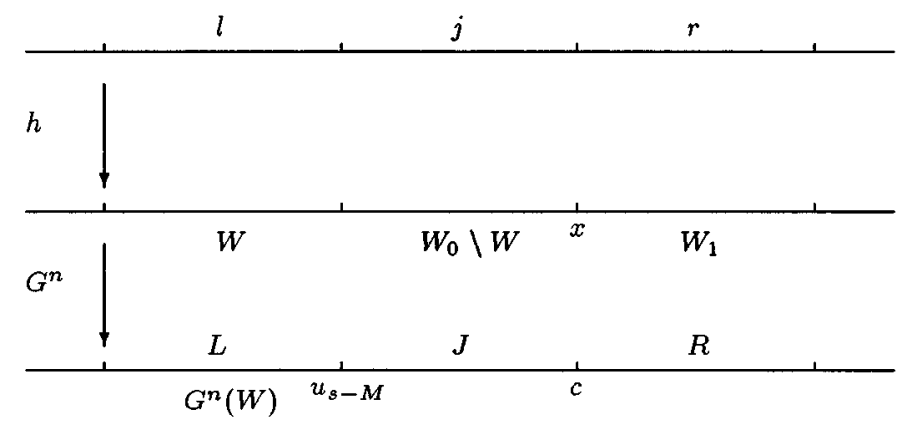

Figure 8.1

Indeed, again let $V \subset U_{\varphi_{0}^{G}, \ldots \varphi_{n-1}^{G}}$ be a branch-domain of $G^{n}$. Assume that $\varphi_{n-1}^{G}(V)=s \geq k_{1}$; then $G^{n}(V)=\left(u_{a}, \hat{u}_{b}\right) \supset\left(u_{s-M-N-1}, \hat{u}_{s-M-N-1}\right)$, whence $G^{n}(V)$ contains an $\varepsilon$-scaled neighbourhood of $\left(u_{s-M}, \hat{u}_{s-M}\right)$. Using (8.3), (8.4) and the choice of $M$,

$$
\begin{aligned}
\mathbb{E}\left(\psi_{n}-\psi_{n-1} \mid V\right) \geq \frac{1}{|V|}\left\{\sum_{r \leq s-M} M\left|V_{r}^{+} \cup V_{r}^{-}\right|-\sum_{r \geq s}(r-s)\left|V_{r}^{+} \cup V_{r}^{-}\right|\right\} \\
\geq M \frac{\left|u_{s-a}-u_{s-M}\right|+\left|\hat{u}_{s-b}-\hat{u}_{s-M}\right|}{\left|u_{a}-\hat{u}_{b}\right|}-\sum_{r \geq s}(r-s) K \frac{\left|u_{r}-\hat{u}_{r}\right|}{\left|u_{a}-\hat{u}_{b}\right|} \\
\geq M \varepsilon-\sum_{r \geq s} K(r-s)(1-\varepsilon)^{\rho(r)-\rho(s)} \\
\geq M \varepsilon-K \sum_{r \geq s}(r-s)(1-\varepsilon)^{\frac{r-s}{N}} \geq 1 .
\end{aligned}
$$

For the variance ( $\leq$ the second moment),

$$
\begin{aligned}
\operatorname{Var}\left(\psi_{n}-\psi_{n-1} \mid V\right) & \leq \frac{1}{|V|}\left\{\sum_{r \leq s} M^{2}\left|V_{r}^{+} \cup V_{r}^{-}\right|+\sum_{r \geq s}(r-s)^{2}\left|V_{r}^{+} \cup V_{r}^{-}\right|\right\} \\
& \leq M^{2}+\sum_{r \geq s}(r-s)^{2} K \frac{\left|u_{r}-\hat{u}_{r}\right|}{\left|u_{a}-\hat{u}_{b}\right|} \\
& \leq M^{2}+\sum_{r \geq s}(r-s)^{2} K(1-\varepsilon)^{\rho(r)-\rho(s)} \\
& \leq M^{2}+K \sum_{r>s}(r-s)^{2}(1-\varepsilon)^{(r-s) / N}<M^{2}+\mathcal{O}\left(\frac{1}{\varepsilon^{5}}\right) .
\end{aligned}
$$

Suppose $f$ has an absorbing Cantor set. Hence by Lemma $5.1, \lim _{n} \varphi_{n}^{G}(x)=$ $\lim _{n} \varphi_{n}(x)=\infty$ a.e. Yet from the above computations and Theorem 5.2, it follows that $\psi_{n} \rightarrow \infty$ on a positive-measured set $X \subset B(\omega(c))$. Therefore for $x \in X$ both $\lim _{n} \varphi_{n}^{G}(x)=\infty$ and $\lim _{n} \varphi_{n}^{G}(x) \leq-\lim _{n} \psi_{n}(x)=-\infty$. This contradiction shows, by means of Lemma 5.1, that $B(\omega(c))$ cannot have full Lebesgue measure.

\section{Absolutely Continuous Invariant Probability Measures}

In this section we give a sufficient condition for the existence of an absolutely continuous (with respect to Lebesgue) invariant probability measure (acip). 


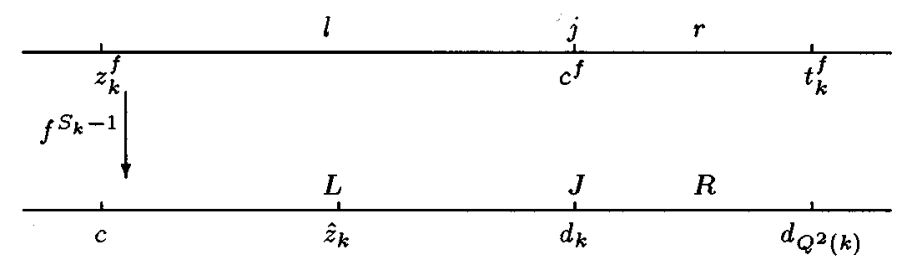

FiguRE 9.1

Theorem 9.1. Let $f$ be non-flat $S$-unimodal. Let there exist $\varepsilon>0$ and $k_{0} \in \mathbb{N}$ such that for all $k \geq k_{0}$

- $\rho_{\varepsilon}(Q(k+1)-1)>\rho_{\varepsilon}\left(Q\left(Q^{2}(k)+1\right)\right)$,

- $\rho_{\varepsilon}(Q(Q(k)+1)-1)>\rho_{\varepsilon}\left(Q\left(Q^{2}(k)+1\right)\right)$, and

- $\sum_{i}(1-\varepsilon)^{\rho_{\varepsilon}(i)-\rho_{\varepsilon}(Q(i+1))}<\infty$.

Then $f$ has an acip.

The first condition on $\rho_{\varepsilon}$ implies that (2.4) holds. Using the results in section 7, we can derive

Corollary 9.2. Let $Q(k)$ be eventually non-decreasing and also $\lim _{k} \frac{k-Q(k)}{\log k}=\infty$. Then every non-flat $S$-unimodal map with kneading map $Q$ has an acip.

Proof of Theorem 9.1. We will prove the theorem by checking that the Nowicki-van Strien summability condition [NS] is satisfied: $f$ has an acip if

$$
\sum_{n=1}^{\infty}\left|D f^{n}\left(c_{1}\right)\right|^{-\frac{1}{\ell}}<\infty
$$

Let $T_{k}$ be the partial sum $\sum_{n=1}^{S_{k}}\left|D f^{n}\left(c^{f}\right)\right|^{-\frac{1}{\ell}}$. Then, by the chain rule,

$$
T_{k+1}=T_{k}+\left|D f^{S_{k}}\left(c^{f}\right)\right|^{-\frac{1}{\ell}} \sum_{n=1}^{S_{Q(k+1)}}\left|D f^{n}\left(d_{k}^{f}\right)\right|^{-\frac{1}{\ell}} .
$$

By the assumptions and Lemma 2.5, there exists $k_{1} \geq k_{0}$ such that for all $k \geq k_{1}$, $f^{S_{k}-1}\left(t_{k}^{f}\right)=d_{Q^{2}(k)}$ and $\left|d_{k}-d_{Q^{2}(k)}\right|>\varepsilon\left|d_{k}-c\right|$.

Using non-flatness and the expansion of cross-ratios, it follows that for $k \geq k_{1}$ (see Figure 9.1)

$$
\begin{aligned}
\left|D f^{S_{k}}\left(c^{f}\right)\right| & =\left|D f\left(d_{k}\right)\right| \cdot\left|D f^{S_{k}-1}\left(c^{f}\right)\right|=\mathcal{O}(\ell)\left|d_{k}-c\right|^{\ell-1} \frac{|J|}{|j|} \\
& \geq \mathcal{O}(\ell)\left|d_{k}-c\right|^{\ell-1} \frac{|t|}{|r|} \frac{|R|}{|T|} \frac{|L|}{|l|} \geq \mathcal{O}(\ell \varepsilon)\left|d_{k}-c\right|^{\ell-1} \frac{\left|d_{k}-c\right|}{\left|z_{k}^{f}-c^{f}\right|} \\
& \geq \mathcal{O}(\ell \varepsilon) \frac{\left|d_{k}-c\right|^{\ell}}{\left|z_{k}^{f}-c^{f}\right|^{\ell}} \geq \mathcal{O}(\ell \varepsilon)(1-\varepsilon)^{\ell\{\rho(Q(k+1))-\rho(k)\}} .
\end{aligned}
$$

Now for the factor $\sum_{n=1}^{S_{Q(k+1)}}\left|D f^{n}\left(d_{k}^{f}\right)\right|^{-\frac{1}{\ell}}$, we compare $\left|D f^{n}\left(d_{k}^{f}\right)\right|$ with $\left|D f^{n}\left(c^{f}\right)\right|$. Again take $k \geq k_{1}$, and consider the points in Figure 9.2.

By assumption

$$
\left|d_{Q^{2}(k+1)}-d_{k+1}\right|,\left|d_{Q^{3}(k+1)}-d_{Q(k+1)}\right| \geq \frac{\varepsilon}{2}\left|d_{k}-d_{Q(k+1)}\right| .
$$




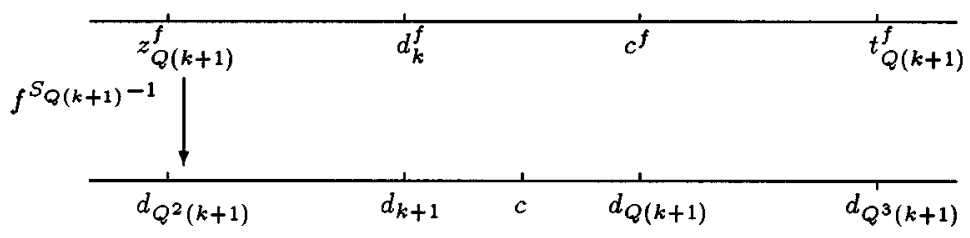

FiguRE 9.2

Hence by the Koebe Principle, there exists $K=\mathcal{O}\left(\frac{1}{\varepsilon^{2}}\right)$ such that

$$
\frac{1}{K} \leq \frac{\left|D f^{S_{Q(k+1)}-1}\left(d_{k}^{f}\right)\right|}{\left|D f^{S_{Q(k+1)}-1}\left(c^{f}\right)\right|} \leq K .
$$

But also for $n<S_{Q(k+1)}$ (because the Koebe spaces are the same),

$$
\frac{1}{K} \leq \frac{\left|D f^{S_{Q(k+1)}-n-1}\left(f^{n}\left(d_{k}^{f}\right)\right)\right|}{\left|D f^{S_{Q(k+1)}-n-1}\left(f^{n}\left(c^{f}\right)\right)\right|} \leq K .
$$

The chain rule gives $\left|D f^{n}\left(d_{k}^{f}\right)\right| \geq \frac{1}{K^{2}}\left|D f^{n}\left(c^{f}\right)\right|$. Combining things, for $k \geq k_{1}$ we get

$$
\begin{aligned}
T_{k+1} & =T_{k}+\left|D f^{S_{k}}\left(c^{f}\right)\right|^{-\frac{1}{\ell}} \sum_{n=1}^{S_{Q(k+1)}}\left|D f^{n}\left(d_{k}^{f}\right)\right|^{-\frac{1}{\ell}} \\
& \leq T_{k}+\mathcal{O}(\ell \varepsilon)(1-\varepsilon)^{\rho(k)-\rho(Q(k+1))} K^{\frac{2}{\ell}} \sum_{n=1}^{S_{Q(k+1)}}\left|D f^{n}\left(c^{f}\right)\right|^{-\frac{1}{\ell}} \\
& \leq T_{k}\left\{1+\mathcal{O}\left(\ell \varepsilon K^{\frac{2}{\ell}}\right)(1-\varepsilon)^{\rho(k)-\rho(Q(k+1))}\right\} .
\end{aligned}
$$

Therefore

$$
\sum_{n \geq 1}\left|D f^{n}\left(c^{f}\right)\right|^{-\frac{1}{\ell}} \leq T_{k_{1}} \prod_{k \geq k_{1}}\left\{1+\mathcal{O}\left(\ell \varepsilon K^{\frac{2}{\ell}}\right)(1-\varepsilon)^{\rho(k)-\rho(Q(k+1))}\right\},
$$

which is finite if $\sum_{k}(1-\varepsilon)^{\rho(k)-\rho(Q(k+1))}<\infty$. This proves Theorem 9.1.

\section{Estimates for FibonacCi-Like Maps}

In this section we give the proofs of (6.3) and (6.4). They will follow from Lemmas 10.6 and 10.7 and non-flatness. The estimates are a generalization of those in [BKNS]. If $Q(k)=\max \{k-2,0\}$, we regain the estimates in that paper.

In this section, $x$ will stand both for the point $x$ as for the distance $|x-c|$. Similarly, $x^{f}$ denotes both the point and the distance $\left|x^{f}-c^{f}\right|$. Let $f$ be an Sunimodal map whose kneading map satisfies $Q(k) \geq k-N$ for some fixed integer $N$. $f$ has no periodic attractor; we assume also that $f$ is not renormalizable. Then Lemmas 2.1 and 2.2 yield that for every $k$ there exists $j_{0} \geq 0$ such that $Q(k+j)=k$ for $0<j<j_{0}$, and $Q\left(k+j_{0}\right)<k$. If $j_{0}$ is large, then we have an almost restrictive interval; cf. [Jo]. One can verify that $\left|c-d_{k}\right|<\left|c-d_{k+1}\right|<\ldots<\left|c-d_{k+j_{0}}\right|$. We also assume (6.1). As $Q(k)<k,(6.1)$ implies (2.4). Define $y_{k}:=f^{S_{Q(k)-1}}\left(d_{k-1}\right)$. Figure 10.1 shows that $y_{k} \in\left(z_{Q^{2}(k)}, \hat{z}_{Q^{2}(k)}\right)$. 


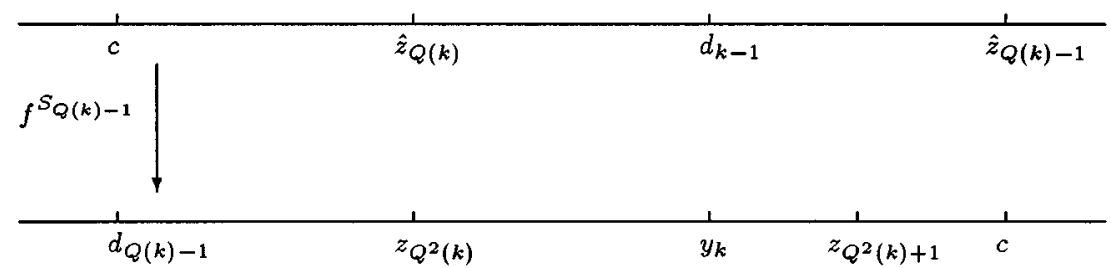

FiguRE 10.1

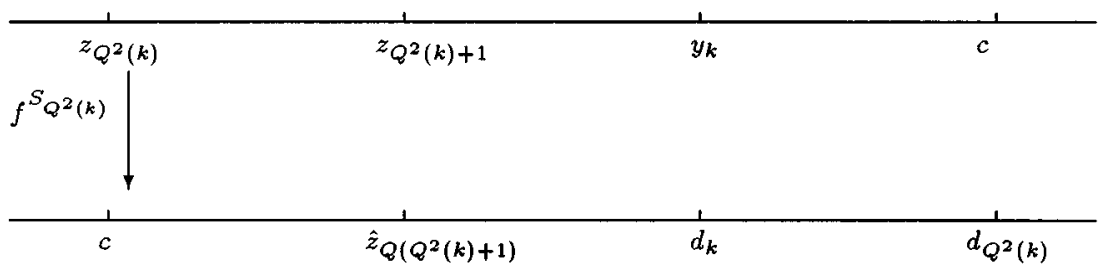

FIGURE 10.2

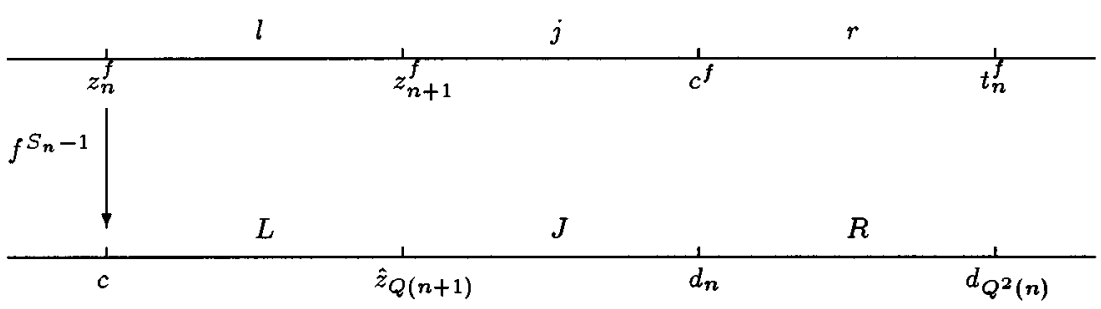

FIGURE 10.3

Lemma 10.1. If (2.4) holds, then for $k$ sufficiently large,

$$
y_{k} \in\left(z_{Q^{2}(k)}, z_{Q^{2}(k)+1}\right) \cup\left(\hat{z}_{Q^{2}(k)+1}, \hat{z}_{Q^{2}(k)}\right) .
$$

Proof. Suppose $(10.1)$ was false, so $y_{k} \in\left(z_{Q^{2}(k)+1}, c\right) \cup\left(c, \hat{z}_{Q^{2}(k)+1}\right)$ (see Figure 10.2).

Then $d_{k}=f^{S_{Q^{2}(k)}}\left(y_{k}\right) \notin\left(z_{Q\left(Q^{2}(k)+1\right)}, \hat{z}_{Q\left(Q^{2}(k)+1\right)}\right)$. Using (2.2) however, we obtain $Q\left(Q^{2}(k)+1\right) \geq Q(k+1)$, violating $(2.4)$.

Lemma 10.2. Let $\Lambda_{n}^{f}:=d_{Q^{2}(n)}^{f} / d_{n}^{f}$. Then for $n$ sufficiently large we have $\Lambda_{n}^{f}>3$ and $\log \Lambda_{n}^{f}>1.1$.

Proof. Pick $m$ arbitrary, and let $n=Q^{2}(m)$.

Consider the intervals as in Figure 10.3. Using non-flatness, expansion of crossratio and the inequality $(\sqrt{a b}-1)^{2} \geq(a-1)(b-1)$, we get 


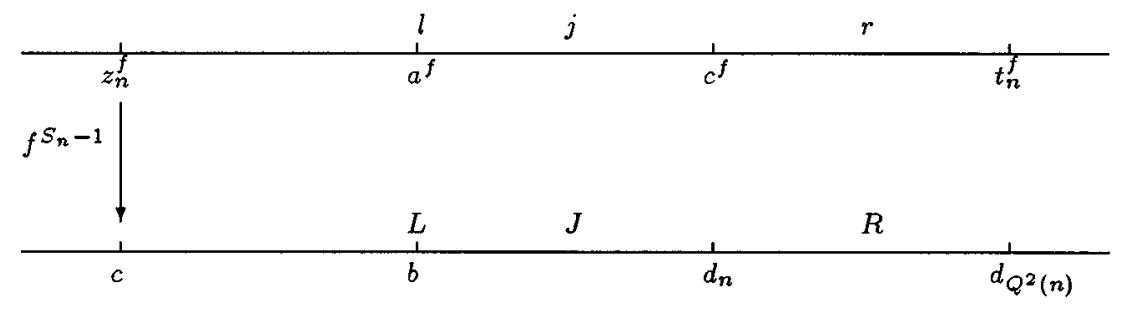

FiguRE 10.4

$$
\begin{aligned}
\frac{1}{\ell}\left(1-\frac{d_{n}^{f}}{d_{Q^{2}(n)}^{f}}\right) & \leq \frac{d_{Q^{2}(n)}-d_{n}}{d_{Q^{2}(n)}}=\frac{|R|}{|T|} \leq \frac{|J|}{|L|} \frac{|l|}{|j|} \\
& =\frac{d_{n}-z_{Q(n+1)}}{z_{Q(n+1)}} \frac{z_{n}^{f}-z_{n+1}^{f}}{z_{n+1}^{f}} \\
& \leq \frac{1}{\ell}\left(\frac{d_{n}^{f}}{z_{Q(n+1)}^{f}}-1\right)\left(\frac{z_{n}^{f}}{z_{n+1}^{f}}-1\right) \\
& \leq \frac{1}{\ell}\left(\sqrt{\frac{d_{n}^{f}}{z_{Q(n+1)}^{f}} \frac{z_{n}^{f}}{z_{n+1}^{f}}}-1\right)^{2} .
\end{aligned}
$$

Hence

$$
\left(1-\frac{d_{n}^{f}}{d_{Q^{2}(n)}^{f}}\right) \leq\left(\sqrt{\frac{d_{n}^{f}}{z_{Q(n+1)}^{f}} \frac{z_{n}^{f}}{z_{n+1}^{f}}}-1\right)^{2} \leq\left(\sqrt{\frac{d_{n}^{f}}{z_{n+1}^{f}}}-1\right)^{2} .
$$

Recall that $Q^{2}(m)=n$. By $(6.1), Q(m+1)>Q^{2}(m)+1=n+1$, so $d_{m}^{f} \in\left(z_{n+1}^{f}, c^{f}\right)$. It follows that

$$
\left(1-\frac{1}{\Lambda_{Q^{2}(m)}^{f}}\right) \leq\left(\sqrt{\frac{d_{n}^{f}}{z_{n+1}^{f}}}-1\right)^{2} \leq\left(\sqrt{\Lambda_{m}^{f}}-1\right)^{2} .
$$

As $1-\frac{1}{x}>(\sqrt{x}-1)^{2}$ for all $x \in(1,3.1), \Lambda_{m}^{f}>\Lambda_{Q^{2}(m)}^{f}$ whenever $\Lambda_{Q^{2}(m)}^{f}<3.1$. Therefore for some sufficiently large $m, \Lambda_{m}^{f}>3.1$. Because $1-\frac{1}{3.1} \leq(\sqrt{x}-1)^{2}$ implies $x \geq 3.1$, it follows that $\Lambda_{m}^{f} \geq 3.1$ for all subsequent $m$.

Lemma 10.3. Let $a \in\left(z_{n}, \hat{z}_{n}\right)$ and $b=f^{S_{n}}(a)$. Then, for $n$ sufficiently large,

$$
\left|D f^{S_{n}}\left(a^{f}\right)\right| \leq \frac{b^{f}}{a^{f}} \log \frac{d_{Q^{2}(n)}^{f}}{b^{f}} \log \frac{d_{n}^{f}}{b^{f}}\left(\frac{d_{Q^{2}(n)}^{f}}{b^{f}}\right)^{\frac{1}{\ell}} .
$$

Proof. We apply non-flatness and the expansion of the cross-ratio on the intervals in Figure 10.4, to get 


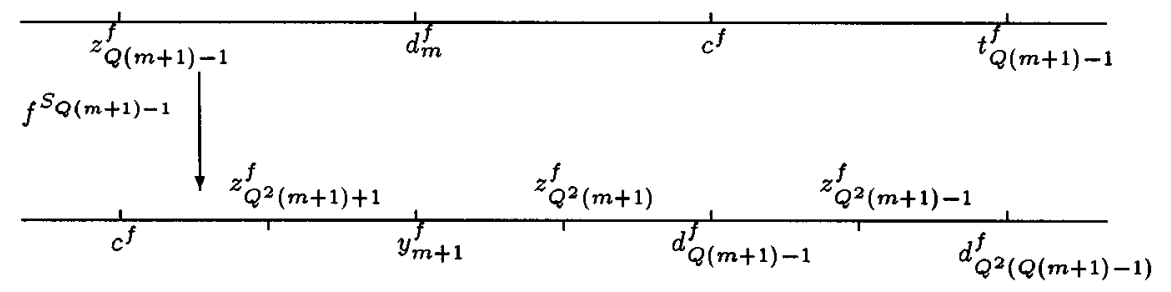

FiguRE 10.5

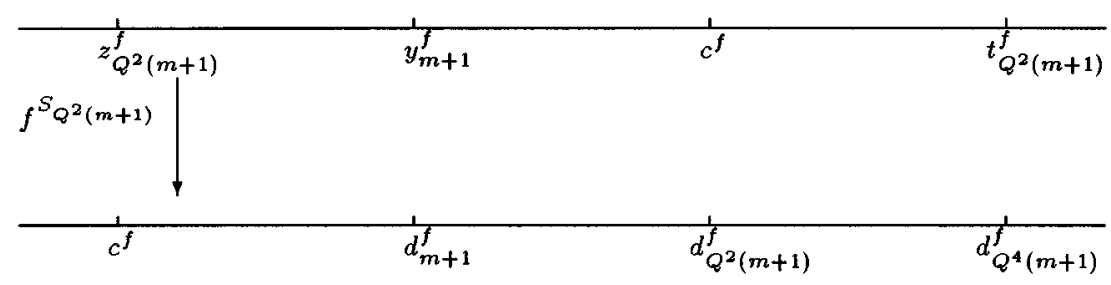

FiguRE 10.6

$$
\begin{aligned}
\left|D f^{S_{n}}\left(a^{f}\right)\right| & \leq 2 \ell \frac{b^{f}}{b} \frac{|L|}{|l|} \leq 2 \ell \frac{b^{f}}{b} \frac{|T|}{|R|} \frac{|J|}{|j|} \frac{|r|}{|t|} \\
& <2 \ell \frac{b^{f}}{b} \frac{d_{Q^{2}(n)}-b}{d_{Q^{2}(n)}-d_{n}} \frac{d_{n}-b}{a^{f}} \\
& =2 \ell \frac{b^{f}}{a^{f}} \frac{d_{n}-b}{d_{n}} \ell \frac{d_{Q^{2}(n)}-b}{d_{Q^{2}(n)}} \frac{1}{\ell \frac{d_{Q^{2}(n)}-d_{n}}{d_{n}}} \frac{d_{Q^{2}(n)}}{b} \\
& \leq \frac{b^{f}}{a^{f}} \frac{2}{\Lambda_{n}^{f}} \log \frac{d_{Q^{2}(n)}^{f}}{b^{f}} \log \frac{d_{n}^{f}}{b^{f}}\left(\frac{d_{Q^{2}(n)}^{f}-d_{n}}{b^{f}}\right)^{\frac{1}{\ell}} .
\end{aligned}
$$

Because $\Lambda_{n}^{f} \geq 3$ for $n$ sufficiently large, the lemma follows.

Define $\mu_{n}^{f}:=\max \left\{z_{k-1}^{f} / z_{k}^{f} \mid n-10 N \leq k \leq n\right\}$.

Lemma 10.4. For $m$ sufficiently large,

$$
\left|D f^{S_{Q(m+1)}}\left(d_{m}^{f}\right)\right| \leq \frac{d_{m+1}^{f}}{d_{m}^{f}} 36 N^{2}(3 N+2) \log ^{4}\left(\mu_{m+1}^{f}\right)\left(\mu_{m+1}^{f}\right)^{\frac{12 N+2}{\ell}} .
$$

Proof. Decompose $\left|D f^{S_{Q(m+1)}}\left(d_{m}^{f}\right)\right|=\left|D f^{S_{Q^{2}(m+1)}}\left(y_{m+1}^{f}\right)\right| \cdot\left|D f^{S_{Q(m+1)-1}}\left(d_{m}^{f}\right)\right|$. For each factor, we will use Lemma 10.3. Consider the second factor, and let $d_{m}^{f}$ take the role of $a^{f}$ (see Figure 10.5). Then

$$
\begin{aligned}
& \left|D f^{S_{Q(m+1)-1}}\left(d_{m}^{f}\right)\right| \\
& \quad \leq \frac{y_{m+1}^{f}}{d_{m}^{f}} \log \frac{d_{Q^{2}(Q(m+1)-1)}^{f}}{y_{m+1}^{f}} \log \frac{d_{Q(m+1)-1}^{f}}{y_{m+1}^{f}}\left(\frac{d_{Q^{2}(Q(m+1)-1)}^{f}}{y_{m+1}^{f}}\right)^{\frac{1}{\ell}} .
\end{aligned}
$$

For the first factor, let $y_{m+1}^{f}$ take the role of $a^{f}$ (Figure 10.6). Then 


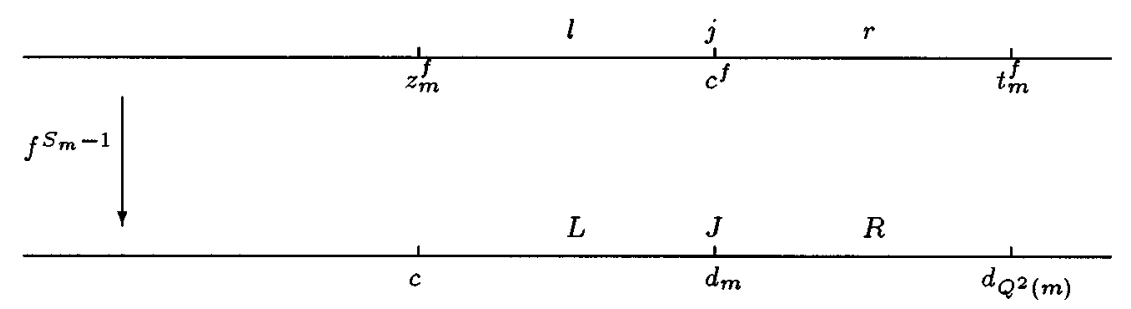

FigURE 10.7

$$
\left|D f^{S_{Q^{2}(m+1)}}\left(y_{m+1}^{f}\right)\right| \leq \frac{d_{m+1}^{f}}{y_{m+1}^{f}} \log \frac{d_{Q^{4}(m+1)}^{f}}{d_{m+1}^{f}} \log \frac{d_{Q^{2}(m+1)}^{f}}{d_{m+1}^{f}}\left(\frac{d_{Q^{4}(m+1)}^{f}}{d_{m+1}^{f}}\right)^{\frac{1}{\ell}} .
$$

From Lemma 10.1 and the definition of $y_{m+1}$, it follows that (see Figure 10.5)

$$
z_{Q^{2}(m+1)+1}^{f}<y_{m+1}^{f}<d_{Q(m+1)-1}^{f}<z_{Q^{2}(m+1)-1}^{f} .
$$

As $Q(n) \geq n-N$, also $\Lambda_{n}^{f} \leq\left(\mu_{n}^{f}\right)^{3 N}$. Combining the above formulas, we get

$$
\begin{aligned}
\mid D f^{S_{Q(m+1)}\left(d_{m}^{f}\right) \mid \leq} & \frac{d_{m+1}^{f}}{d_{m}^{f}} \cdot \log \left(\frac{z_{Q^{2}(m+1)-1}^{f}}{z_{Q^{2}(m+1)+1}^{f}}\right) \\
& \cdot \log \left(\frac{z_{Q^{2}(m+1)-1}^{f}}{z_{Q^{2}(m+1)+1}^{f}} \Lambda_{Q(m+1)-1}^{f}\right)\left(\frac{z_{Q^{2}(m+1)-1}^{f}}{z_{Q^{2}(m+1)+1}^{f}} \Lambda_{Q(m+1)-1}^{f}\right)^{\frac{1}{\ell}} \\
& \cdot \log \left(\Lambda_{m+1}^{f}\right) \log \left(\Lambda_{m+1}^{f} \Lambda_{Q^{2}(m+1)}^{f}\right)\left(\Lambda_{m+1}^{f} \Lambda_{Q^{2}(m+1)}^{f}\right)^{\frac{1}{\ell}} \\
\leq & \frac{d_{m+1}^{f}}{d_{m}^{f}} 36 N^{2}(3 N+2) \log ^{4}\left(\mu_{m+1}^{f}\right)\left(\mu_{m+1}^{f}\right)^{\frac{12 N+2}{\ell}}
\end{aligned}
$$

as asserted.

Lemma 10.5. For $m$ sufficiently large,

$$
\frac{d_{m}^{f}}{z_{m}^{f}}\left(\frac{d_{m}^{f}}{d_{Q^{2}(m)}^{f}}\right)^{\frac{1}{\ell}} \leq\left|D f^{S_{m}}\left(c^{f}\right)\right| \leq 2 \frac{d_{m}^{f}}{z_{m+1}^{f}} \log \frac{d_{m}^{f}}{z_{Q(m+1)}^{f}} .
$$

Proof. For the first inequality, we use the expansion of the cross-ratio of the intervals in Figure 10.7, and get

$$
\begin{aligned}
\left|D f^{S_{m}}\left(c^{f}\right)\right| & \geq 0.95 \ell \frac{d_{m}^{f}}{d_{m}} \frac{|J|}{|j|} \geq 0.95 \ell \frac{d_{m}^{f}}{d_{m}} \frac{|L|}{|T|} \frac{|R|}{|l|} \frac{|t|}{|r|} \geq 0.95 \ell \frac{d_{m}^{f}}{d_{m}} \frac{d_{m}}{d_{Q^{2}(m)}} \frac{d_{Q^{2}(m)}-d_{m}}{z_{m}^{f}} \\
& \geq 0.95 \frac{d_{m}^{f}}{z_{m}^{f}}\left(\frac{d_{m}^{f}}{d_{Q^{2}(m)}^{f}}\right)^{\frac{1}{\ell}} \log \Lambda_{m}^{f} \geq \frac{d_{m}^{f}}{z_{m}^{f}}\left(\frac{d_{m}^{f}}{d_{Q^{2}(m)}^{f}}\right)^{\frac{1}{\ell}},
\end{aligned}
$$




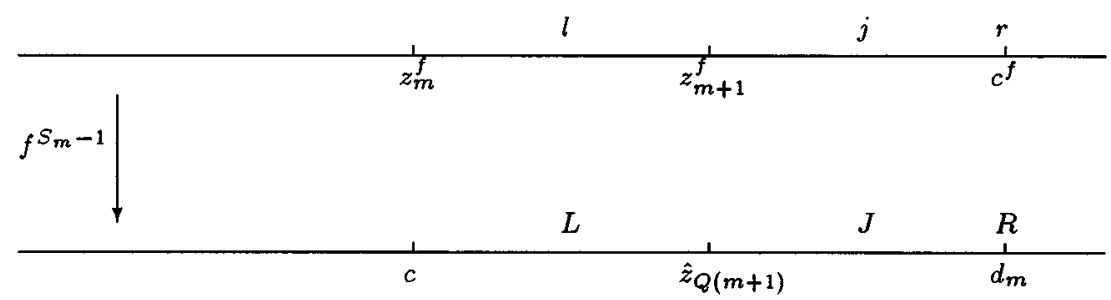

FigURE 10.8

where we have used Lemma 10.2 for the last step. For the second inequality, we use the intervals in Figure 10.8, and get

$$
\begin{aligned}
\left|D f^{S_{m}}\left(c^{f}\right)\right| & \leq \sqrt{2} \ell \frac{d_{m}^{f}}{d_{m}} \frac{|R|}{|r|} \leq \sqrt{2} \ell \frac{d_{m}^{f}}{d_{m}} \frac{|T|}{|L|} \frac{|J|}{|j|} \frac{|l|}{|t|} \\
& \leq \sqrt{2} \ell \frac{d_{m}^{f}}{d_{m}} \frac{d_{m}}{z_{Q(m+1)}} \frac{d_{m}-z_{Q(m+1)}}{z_{m+1}^{f}} \frac{z_{m}^{f}-z_{m+1}^{f}}{z_{m}^{f}} \\
& \leq 2 \frac{d_{m}^{f}}{z_{m+1}^{f}} \log \frac{d_{m}^{f}}{z_{Q(m+1)}^{f}} .
\end{aligned}
$$

This proves the lemma.

Lemma 10.6. $D f^{S_{n}}\left(c^{f}\right)$ is bounded and bounded away from 0 . Moreover, $\frac{z_{n-1}^{f}}{z_{n}^{f}}$ is bounded.

Proof. We first prove that $\frac{z_{n-1}^{f}}{z_{n}^{f}}$ is bounded. Then we use Lemma 10.5 to prove the first statement.

Take $n$ such that $z_{n-1}<d_{n}$, i.e. $Q(n+1)<n$. Next take $M$ minimal such that $z_{n-M+1}>d_{n}$. As $Q(n+1) \geq n-N+1$, it follows that $M \leq N$. Decompose $D f^{S_{n}}\left(c^{f}\right)$ into $M$ factors as follows:

$$
\begin{aligned}
D f^{S_{n}}\left(c^{f}\right) & =D f^{S_{n-1}}\left(c^{f}\right) \cdot D f^{S_{Q(n)}}\left(d_{n-1}^{f}\right) \\
& =D f^{S_{n-2}}\left(c^{f}\right) \cdot D f^{S_{Q(n-1)}}\left(d_{n-2}^{f}\right) \cdot D f^{S_{Q(n)}}\left(d_{n-1}^{f}\right) \\
\vdots & \vdots \\
& =D f^{S_{n-M}}\left(c^{f}\right) \cdot D f^{S_{Q(n-M+1)}}\left(d_{n-M}^{f}\right) \cdots D f^{S_{Q(n)}}\left(d_{n-1}^{f}\right) .
\end{aligned}
$$

We apply Lemma 10.5 to the first factor and Lemma 10.4 to the other factors. 


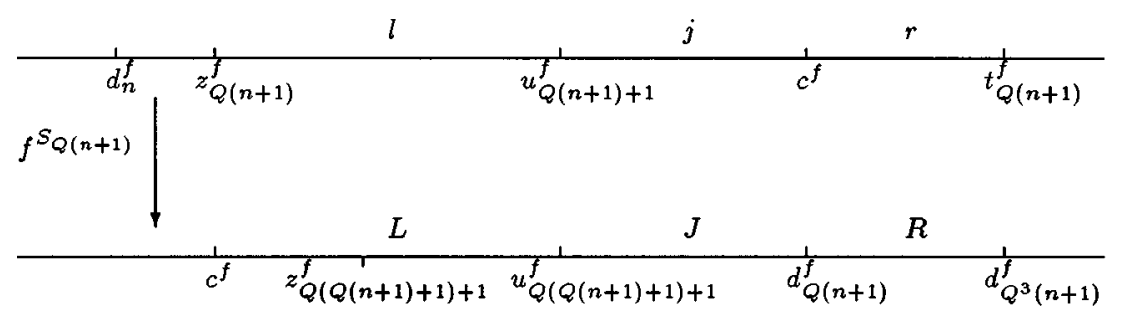

FiguRe 10.9

$$
\begin{aligned}
\left|D f^{S_{n}}\left(c^{f}\right)\right| \leq & 2 \frac{d_{n-M}^{f}}{z_{n-M+1}^{f}} \log \frac{d_{n-M}^{f}}{z_{Q(n-M+1)}^{f}} \\
& \cdot \frac{d_{n-M+1}^{f}}{d_{n-M}^{f}} 36 N^{2}(3 N+2) \log ^{4}\left(\mu_{n-M+1}^{f}\right)\left(\mu_{n-M+1}^{f}\right)^{\frac{12 N+2}{\ell}} \\
& \vdots \\
& \cdot \frac{d_{n-1}^{f}}{d_{n-2}^{f}} 36 N^{2}(3 N+2) \log ^{4}\left(\mu_{n-1}^{f}\right)\left(\mu_{n-1}^{f}\right)^{\frac{12 N+2}{\ell}} \\
& \cdot \frac{d_{n}^{f}}{d_{n-1}^{f}} 36 N^{2}(3 N+2) \log ^{4}\left(\mu_{n}^{f}\right)\left(\mu_{n}^{f}\right)^{\frac{12 N+2}{\ell}} \\
\leq & \cdot 36^{M} N^{2 M}(3 N+2)^{M} \log ^{4 M+1}\left(\mu_{n}^{f}\right)\left(\mu_{n}^{f}\right)^{\frac{(12 N+2) M}{\ell}}
\end{aligned}
$$

As $M \leq N$, and by the first part of Lemma 10.5,

$$
\frac{d_{n}^{f}}{z_{n}^{f}} \leq 2 \cdot 36^{N} N^{2 N}(3 N+2)^{N} \log ^{4 N+1}\left(\mu_{n}^{f}\right)\left(\mu_{n}^{f}\right)^{\frac{(12 N+2) N+1}{\ell}} .
$$

If $Q(n+1)<n$, then $\frac{z_{n-1}^{f}}{z_{n}^{f}} \leq \frac{d_{n}^{f}}{z_{n}^{f}}$. If $Q(n+1)=n$, then, as $f$ is not renormalizable (see Lemma 2.3) and $Q(k)>k-N$, there exists $j_{0} \leq N$ such that $Q(n+j)=n$ for $0<j<j_{0}$, and $Q\left(n+j_{0}\right)<n$. Hence $\frac{z_{n-1}^{f}}{z_{n}^{f}} \leq \frac{d_{n+j_{0}}^{f}}{z_{n+j_{0}}^{f}}$, and for $\mu_{\infty}^{f}=\lim \sup _{n} \mu_{n}^{f}$,

$$
\mu_{\infty}^{f} \leq 2 \cdot 36^{N} N^{2 N}(3 N+2)^{N} \log ^{4 N+1}\left(\mu_{\infty}^{f}\right)\left(\mu_{\infty}^{f}\right)^{\frac{(12 N+2) N+1}{\ell}} .
$$

Hence for $\ell$ sufficiently large, $\mu_{\infty}^{f}<\infty$.

In general, $z_{Q(n+1)}<d_{n}<d_{Q^{2}(n)}<z_{Q\left(Q^{2}(n)+1\right)-1} \leq z_{Q(n+1)-3 N}$ and $z_{Q(n+1)-1}$ $>d_{n}>z_{n-N}$. It follows by Lemma 10.5 that

$$
0<\left(\mu_{\infty}^{f}\right)^{-\frac{3 N}{\ell}} \leq\left|D f^{S_{m}}\left(c^{f}\right)\right| \leq\left(\mu_{\infty}^{f}\right)^{N} \log \mu_{\infty}^{f},
$$

for $m$ sufficiently large.

Lemma 10.7. $\frac{d_{n}^{f}}{u_{Q(n+1)+1}^{f}} \geq K>1$ for $n$ sufficiently large.

Proof. We will use the expansion of the cross-ratio for the intervals in Figure 10.9, and get 


$$
\begin{aligned}
\frac{d_{n}^{f}-u_{Q(n+1)+1}^{f}}{u_{Q(n+1)+1}^{f}} & \geq \frac{z_{Q(n+1)}^{f}-u_{Q(n+1)+1}^{f}}{u_{Q(n+1)+1}^{f}}=\frac{|l|}{|j|} \geq \frac{|L|}{|T|} \frac{|R|}{|J|} \mid \frac{|t|}{|r|} \\
& \geq \frac{u_{Q(Q(n+1)+1)+1}^{f}}{d_{Q^{3}(n+1)}^{f}} \frac{d_{Q^{3}(n+1)}^{f}-d_{Q(n+1)}^{f}}{d_{Q(n+1)}^{f}-u_{Q(Q(n+1)+1)+1}^{f}} \\
& \geq \frac{u_{Q(Q(n+1)+1)+1}^{f}}{d_{Q(n+1)}^{f}}\left(1-\frac{d_{Q(n+1)}^{f}}{d_{Q^{3}(n+1)}^{f}}\right) \\
& \geq \frac{u_{Q(Q(n+1)+1)+1}^{f}}{d_{Q(n+1)}^{f}}\left(1-\frac{1}{\Lambda_{Q(n+1)}^{f}}\right) .
\end{aligned}
$$

Now

$$
\frac{u_{Q(Q(n+1)+1)+1}^{f}}{d_{Q(n+1)}^{f}} \geq \frac{z_{Q(Q(n+1)+1)+1}^{f}}{z_{Q(Q(n+1)+1)-1}^{f}} \geq \frac{1}{\left(\mu_{\infty}^{f}\right)^{2}},
$$

and by Lemma $10.2, \Lambda_{Q(n+1)}^{f} \geq 3$. Therefore by Lemma 10.6

$$
\frac{d_{n}^{f}}{u_{Q(n+1)+1}^{f}}-1=\frac{d_{n}^{f}-u_{Q(n+1)+1}^{f}}{u_{Q(n+1)+1}^{f}} \geq \frac{2}{3} \frac{1}{\left(\mu_{\infty}^{f}\right)^{2}}>0 .
$$

This proves Lemma 10.7 .

\section{Some Counterexamples}

In this section we give the counterexamples which complete the proof of Proposition 3.1 .

Example 11.1. This example gives the kneadings of a unimodal map satisfying the property $(\mathrm{C} 7)$ (which is equivalent to $r_{n}\left(c_{1}\right) \rightarrow 0$ ), but failing condition $(\mathrm{C} 6)$. More precisely, we construct the kneadings of a map such that

- $M_{n}\left(c_{1}\right) \supset\left(z_{0}, \hat{z}_{0}\right) \ni c_{n+1}$ infinitely often, and $d\left(c_{n+1},\left\{z_{0}, \hat{z}_{0}\right\}\right) \rightarrow 0$ for these values of $n$.

- For every $\varepsilon>0$ there exists only finitely many $n$ 's such that $M_{n}\left(c_{1}\right) \supset$ $\left(z_{0}-\varepsilon, \hat{z}_{0}+\varepsilon\right)$.

- For those $n$ for which $M_{n}\left(c_{1}\right) \ni c$ but $M_{n}\left(c_{1}\right) \not \supset\left(z_{0}, z_{0}\right), d\left(c, \partial M_{n}\left(c_{1}\right)\right) \rightarrow 0$.

Start with $Q(0)=Q(1)=Q(2)=0$, and $k_{0}=1$ and $k_{1}=3$. Then we define, inductively,

$$
\begin{aligned}
& Q\left(k_{i}\right)=k_{i-1}, \\
& Q\left(k_{i}+1\right)=0, \\
& Q\left(k_{i}+2\right)=k_{i-1}+2, \\
& Q\left(k_{i}+3\right)=k_{i}+2, \\
& Q\left(k_{i}+4\right)=k_{i}, \\
& Q\left(k_{i}+5\right)=1, \\
& Q\left(k_{i}+5+j\right)=Q(j) \text { for } j=1,2, \ldots, k_{i-1}+3, \\
& \text { and } \\
& k_{i+1}=k_{i}+k_{i-1}+9 .
\end{aligned}
$$


Example 11.2. We construct the kneading map of a unimodal map having the following properties:

- $R_{n}\left(c_{1}\right) \nrightarrow 0$,

- $d\left(c, \partial M_{n}\left(c_{1}\right)\right) \rightarrow 0$ as $n \rightarrow \infty$, and $M_{n}\left(c_{1}\right) \ni c$.

In other words, if $M_{n}\left(c_{1}\right) \ni c$, then at least one end-point of $M_{n}\left(c_{1}\right)$ must be close to $c$ for $n$ large. Therefore condition (C5) is satisfied, but (C3), or equivalently (C4), fails. The kneading map is as follows: $Q(0)=Q(1)=0$ and, for $k>1$,

$$
Q(k)=\left\{\begin{array}{lll}
0 & \text { if } k \equiv 0 \quad \bmod 3, \\
k-2 & \text { if } k \equiv 1 \quad \bmod 3 \\
k-1 & \text { if } k \equiv 2 & \bmod 3 .
\end{array}\right.
$$

Example 11.3. We construct the kneading map of a unimodal map that satisfies condition (C6), but fails (C5). It has the following properties:

- There exist infinitely many $n$ 's such that $M_{n}\left(c_{1}\right) \supset\left(z_{0}, \hat{z}_{0}\right)$ and $c_{n+1} \notin\left[z_{0}, \hat{z}_{0}\right]$. (Notice that in Example 11.1, $c_{n+1} \in\left(z_{0}, \hat{z}_{0}\right)$ for the comparable iterates.)

- For every $\varepsilon>0$ there exist only finitely many $n$ 's such that $M_{n}\left(c_{1}\right) \supset$ $\left(z_{0}-\varepsilon, \hat{z}_{0}+\varepsilon\right)$.

- For those $n$ for which $M_{n}\left(c_{1}\right) \ni c$ but $M_{n}\left(c_{1}\right) \not \supset\left(z_{0}, z_{0}\right), d\left(c, \partial M_{n}\left(c_{1}\right)\right) \rightarrow 0$ as $n \rightarrow \infty$.

The kneading map is as follows: $Q(0)=Q(1)=0$, and for $k>1$,

$$
Q(k)=\left\{\begin{array}{lll}
0 & \text { if } k \equiv 0 \bmod 4 \text { or } k \equiv 3 \bmod 4, \\
k-3 & \text { if } k \equiv 1 \bmod 4, \\
k-1 & \text { if } k \equiv 2 \bmod 4 .
\end{array}\right.
$$

Before we can prove that the examples really do what they are supposed to, we have to discuss a few properties of cutting and co-cutting times. For more details we refer to [B2], [B3]. We will mainly consider the combinatorial side of these notions. Let $\nu=e_{1} e_{2} e_{3} e_{4} \ldots=10 e_{3} e_{4} \ldots$ be the kneading invariant. The cutting times can be found by decomposing $\nu$ into blocks that repeat the head of $\nu$. Therefore $S_{0}=1$ and $S_{k}=\min \left\{j>S_{k-1} \mid e_{j} \neq e_{j-S_{k-1}}\right\}$. Writing $0^{\prime}=1$ and $1^{\prime}=0$, we get

$$
e_{S_{k-1}+1} e_{S_{k-1}+2} \ldots e_{S_{k}-1} e_{S_{k}}=e_{1} e_{2} \ldots e_{S_{Q(k)}} e_{S_{Q(k)}}^{\prime},
$$

where $Q$ is as ever the kneading map. Recall that admissibility in terms of the kneading map is guaranteed by

$$
\{Q(k+j)\}_{j \geq 1} \succeq\left\{Q\left(Q^{2}(k)+j\right)\right\}_{j \geq 1} .
$$

The co-cutting times can be found using the other splitting: $T_{0}=\min \left\{j>1 \mid e_{j}=\right.$ $1\}$, and $T_{k}=\min \left\{j>T_{k-1} \mid e_{j} \neq e_{j-T_{k-1}}\right\}$. Hence

$$
e_{T_{k-1}+1} e_{T_{k-1}+2 \ldots} e_{T_{k}-1} e_{T_{k}}=e_{1} e_{2} \ldots e_{S_{\tilde{Q}(k)}} e_{S_{\tilde{Q}(k)}}^{\prime} .
$$

Define

$$
S\langle n\rangle:=\max \left\{S_{j} \mid S_{j}<n\right\}, \quad T\langle n\rangle:=\max \left\{T_{j} \mid T_{j}<n\right\} .
$$

Geometrically speaking, $M_{n-1}\left(c_{1}\right) \ni c$ if and only if $n$ is a cutting or co-cutting time. The iterate $n$ is a cutting time if $f^{n-1}\left(H_{n}\left(c_{1}\right) \cap\left(0, c_{1}\right)\right) \ni c$, and $n$ is a cocutting time if $f^{n-1}\left(H_{n}\left(c_{1}\right) \backslash\left(0, c_{1}\right)\right) \ni c$. One can prove that $T_{l}-S\left\langle T_{l}\right\rangle$ is always 
a cutting time. (In fact, will prove this inductively in these heuristics.) Suppose that $S_{k-1}=S\left\langle T_{l}\right\rangle$, and that $T_{l}-S\left\langle T_{l}\right\rangle=S_{r}$; then (11.1) and (11.2) yield

$$
\begin{array}{cc}
e_{S_{k-1}+1} e_{S_{k-1}+2} \ldots e_{T_{l}} \ldots e_{T_{l+1}} \ldots & \ldots e_{S_{k}-1} e_{S_{k}} \\
=e_{1} e_{2} \ldots e_{S_{r}} \ldots e_{S_{r+1}} \ldots & \ldots e_{S_{Q(k)}-1} e_{S_{Q(k)}}^{\prime}
\end{array} .
$$

This implies that for the subsequent co-cutting times $T_{l}, T_{l+1}, \ldots$ (as long as they are smaller than $\left.S_{k}\right), T_{l}-S_{k-1}=S_{r}, T_{l+1}-S_{k-1}=S_{r+1}, \ldots$ In particular, if $T_{l^{\prime}}=T\left\langle S_{k}\right\rangle$, then

$$
T_{l^{\prime}}=S_{k-1}+S_{Q(k)-1}=S_{k}-S_{Q^{2}(k)},
$$

and between $S_{k}-S_{Q^{2}(k)}$ and $S_{k}$ there are no co-cutting times.

In order to find the co-cutting time $T_{l^{\prime}+1}$, we have to take a closer look at (2.3). Let $\beta(k)>0$ be such that $\left.Q(k+j)=Q\left(Q^{2}(k)+j\right)\right)$ for $0<j<\beta(k)$, and $Q(k+\beta(k))>Q\left(Q^{2}(k)+\beta(k)\right)$. Hence $S_{k+j}=S_{Q^{2}(k)+j}$ for all $j<\beta(k)$, and $S_{k+\beta(k)}>S_{Q^{2}(k)+\beta(k)}$. Therefore

$$
\begin{aligned}
e_{S_{k}-S_{Q^{2}(k)+1}} \ldots e_{S_{k}} \ldots e_{S_{k+1}} \ldots \quad \ldots e_{S_{k+\beta(k)-1}} \ldots e_{S_{k+\beta(k)-1}+S_{n}} \ldots e_{S_{k+\beta(k)}} & \\
& =e_{1} \ldots e_{S_{Q^{2}(k)}} \ldots e_{S_{Q^{2}(k)+1}} \ldots \quad \ldots e_{S_{Q^{2}(k)+\beta(k)-1}} \ldots e_{S_{Q^{2}(k)+\beta(k)}}^{\prime} e_{1} e_{2} \ldots,
\end{aligned}
$$

where $S_{n}=S_{Q^{2}(k)+\beta(k)}-S_{Q^{2}(k)+\beta(k)-1}=S_{Q\left(Q^{2}(k)+\beta(k)\right)}$. Therefore

$$
T_{l^{\prime}+1}=T_{l^{\prime}}+S_{Q^{2}(k)+\beta(k)},
$$

and

$$
T_{l^{\prime}+1}-S_{k+\beta(k)-1}=T_{l^{\prime}+1}-S\left\langle T_{l^{\prime}+1}\right\rangle=S_{Q\left(Q^{2}(k)+\beta(k)\right)} .
$$

Lemma 11.1. Suppose $R_{n}\left(c_{1}\right) \geq \varepsilon$. Then there exists $K=K(\varepsilon)$ such that $\{n+$ $1, \ldots, n+K\}$ contains a cutting OR a co-cutting time. If $r_{n}\left(c_{1}\right) \geq \varepsilon$, then $\{n+$ $1, \ldots, n+K\}$ contains a cutting AND a co-cutting time. In particular, this holds if $n+1$ is a cutting or co-cutting time.

Proof. Follows immediately from the Contraction Principle.

The reverse need not be true. If $M_{n}\left(c_{1}\right) \ni z_{k}$, then $n+S_{k}$ is a cutting or a co-cutting time, but it gives no information on the size of $M_{n}\left(c_{1}\right)$. However, if $Q(k)=r$, then $M_{S_{k-1}-1}\left(c_{1}\right) \subset\left(c, z_{r-1}\right)$; likewise if $\tilde{Q}(l)=r$.

The next corollary is straightforward.

Corollary 11.2. Let $S_{+}\langle n\rangle:=\min \left\{S_{k} \mid S_{k}>n\right\}$ and $T_{+}\langle n\rangle:=\min \left\{T_{l} \mid T_{l}>n\right\}$. If

$$
\lim _{n \in\left\{S_{k}\right\} \cup\left\{T_{l}\right\}, n \rightarrow \infty} \max \left\{S_{+}\langle n\rangle-n, T_{+}\langle n\rangle-n\right\}=\infty,
$$

then $r_{n-1}\left(c_{1}\right) \rightarrow 0$.

This gives us enough luggage to prove that the examples do what they are supposed to do.

Proof of Example 11.1. We first check (2.3).

i $r=k_{i}$. Then $Q(r+1)=0=Q\left(k_{i-2}+1\right)=Q\left(Q^{2}(r)+1\right)$ and $Q\left(k_{i}+2\right)=$ $k_{i-1}+2>k_{i-3}+2=Q\left(Q^{2}(r)+2\right)$.

ii $r=k_{i}+1$. Then $Q(r+1)=k_{i-1}+2>0=Q(1)=Q\left(Q^{2}(r)+1\right)$.

iii $r=k_{i}+2$. Then $Q(r+1)=k_{i}+2>k_{i-2}+2=Q\left(Q^{2}(r)+1\right)$.

iv $r=k_{i}+3$. Then $Q(r+1)=k_{i}>0=Q\left(k_{i}+1\right)=Q\left(Q^{2}(r)+1\right)$. 
$\mathrm{v} r=k_{i}+4$. Then $Q(r+1)=1>0=Q\left(k_{i-1}+1\right)=Q\left(Q^{2}(r)+1\right)$.

vi $r=k_{i}+5$. By construction, $Q(r+j)=Q(j)=Q\left(Q^{2}(r)+j\right)$ for $j=$ $1, \ldots, k_{i-1}+3$.

But $Q\left(r+k_{i-1}+4\right)=Q\left(k_{i+1}\right)=k_{i}>k_{i-1}=Q\left(Q^{2}(r)+k_{i-1}+4\right)$. This settles admissibility.

We will show that $M_{n}\left(c_{1}\right) \supset\left(z_{0}, \hat{z}_{0}\right) \ni c_{n+1}$ infinitely often. Let

$$
a_{n}:=f^{n-1}\left(\partial H_{n-1}\left(c_{1}\right) \cap(0, c)\right), \quad b_{n}:=f^{n-1}\left(\partial H_{n-1}\left(c_{1}\right) \backslash(0, c)\right) .
$$

Hence $\left(a_{n}, b_{n}\right)=M_{n-1}\left(c_{1}\right)$. Abbreviate $r=k_{i}+4$. Then $Q(r+1)=1$, so $c_{S_{r}} \in A_{1} \cup \hat{A}_{1}$. Also $a_{S_{r}}=c_{S_{Q(r)}}=c_{S_{k_{i}}}$, and, as $Q\left(k_{i}+1\right)=0, a_{S_{r}} \in A_{0} \cup \hat{A}_{0}$. The other endpoint $b_{S_{r}}=c_{S_{Q^{2}(r)}}=c_{S_{k_{i-1}}}$. As $Q\left(k_{i-1}+1\right)=0$, also $b_{S_{r}} \in A_{0} \cup \hat{A}_{0}$. Hence $M_{S_{r}-1}\left(c_{1}\right) \supset\left(z_{0}, \hat{z}_{0}\right) \ni c_{S_{r}}$ for every $i$. In particular $R_{S_{r}-1}\left(c_{1}\right) \nrightarrow 0$.

However, $\left|c_{S_{r}}-b_{S_{r}}\right| \rightarrow 0$, as we shall prove. It follows by (11.3) that $T_{+}\left\langle S_{r}\right\rangle=$ $S_{r}+S_{Q\left(Q^{2}(r)+1\right)}=S_{r}+1$. By construction of $Q$,

$$
\begin{aligned}
T_{+}\left\langle S_{r}+1\right\rangle= & S_{r+1}+S_{Q\left(Q^{2}(r+1)+1\right)} \\
& \quad+\ldots+S_{Q\left(Q^{2}(r+1)+k_{i-1}+3\right)}+S_{Q\left(Q^{2}(r+1)+k_{i-1}+4\right)} \\
= & S_{k_{i}+k_{i-1}+8}+S_{k_{i-1}} .
\end{aligned}
$$

Hence $T_{+}\left\langle S_{r}+1\right\rangle-\left(S_{r}+1\right) \rightarrow \infty$ and $f\left(c_{S_{r}}\right) \rightarrow c$ as $i \rightarrow \infty$.

On the other hand, $b_{S_{r}}=c_{S_{Q^{2}(r)}}=c_{S_{k_{i-1}}}$ and $f\left(b_{S_{r}}\right)=c_{S_{k_{i-1}+1}}=c_{S_{k_{i-1}+1}}$. Then $Q\left(k_{i-1}+2\right)=k_{i-2}+2 \rightarrow \infty$, and therefore also $f\left(b_{S_{r}}\right) \rightarrow c$ as $i \rightarrow \infty$.

Combining these facts, we get $\left|c_{S_{r}}-b_{S_{r}}\right| \rightarrow 0$, as asserted.

Finally we have to check that $r_{n}\left(c_{1}\right) \rightarrow 0$. Due to the Contraction Principle, it suffices to consider the cutting and co-cutting times only. Let us start with the cutting times:

vii $r=k_{i}$. Then $T\left\langle S_{r}\right\rangle=S_{r}-S_{Q^{2}(r)}=S_{r}-S_{k_{i-2}}, T_{+}\left\langle S_{r}\right\rangle=S_{r}+S_{Q\left(Q^{2}(r)+1\right)}+$ $S_{Q\left(Q^{2}(r)+2\right)}=S_{r}+1+S_{k_{i-3}+2}$. Therefore $T_{+}\left\langle S_{r}\right\rangle-S_{r}=1+S_{k_{i-3}+2} \rightarrow \infty$ and $\left|b_{S_{r}}-c_{S_{r}}\right| \rightarrow 0$ as $i \rightarrow \infty$.

viii $r=k_{i}+1$. Similar to vii.

ix $r=k_{i}+2 . \quad S_{r}-S\left\langle S_{r}\right\rangle=S_{Q(r)}=S_{k_{i-1}+2}$. Because $Q\left(k_{i-1}+2+1\right)=$ $k_{i-1}+2 \rightarrow \infty$ as $i \rightarrow \infty$, also $a_{S_{r}} \rightarrow c$ as $i \rightarrow \infty$. Because $Q(r+1)=k_{i}+2 \rightarrow \infty$, also $c_{S_{r}} \rightarrow c$ and therefore $\left|a_{S_{r}}-c_{S_{r}}\right| \rightarrow c$ as $i \rightarrow \infty$.

$\mathrm{x} r=k_{i}+3$. Similar to ix.

xi $r=k_{i}+4$. This case we treated above.

xii $r=k_{i}+4+j$ for $j=0,1, \ldots, k_{i-1}+3$. From (11.5) it follows that $T_{+}\left\langle S_{r}\right\rangle=$ $S_{k_{i}+k_{i-1}+8}+S_{k_{i-1}}$. Therefore $T_{+}\left\langle S_{r}\right\rangle-S_{r} \geq S_{k_{i-1}} \rightarrow \infty$ and $b_{S_{r}} \rightarrow c_{S_{r}}$ as $i \rightarrow \infty$. Finally, we have to look after the co-cutting times.

0 .

xiii $S_{k_{i}-1}<T_{l}<S_{k_{i}}$. Then $S_{+}\left\langle T_{l}\right\rangle-T_{l} \geq S_{Q^{2}\left(k_{i}\right)}=S_{k_{i-2}} \rightarrow \infty$ and $\left|a_{T_{l}}-c_{T_{l}}\right| \rightarrow$

xiv $S_{k_{i}}<T_{l}<S_{k_{i}+1}$ is impossible, because $Q\left(k_{i}+1\right)=0$.

xv $S_{k_{i}+1}<T_{l}<S_{k_{i}+2}$. See xiii.

xvi $S_{k_{i}+2}<T_{l}<S_{k_{i}+3}$. See xiii.

xvii $S_{k_{i}+3}<T_{l}<S_{k_{i}+4}$. See xiii.

xviii $T_{l}=S_{k_{i}+4}+1$. Then $T_{l-1}=S_{k_{i}+4}-S_{Q^{2}\left(k_{i}+4\right)}=S_{k_{i}+4}-S_{k_{i-1}}$. Therefore $T_{l}-T_{l-1}=S_{\tilde{Q}(l)}=S_{k_{i-1}}+1=S_{k_{i-1}+1}$. Because $Q(\tilde{Q}(l)+1)=Q\left(k_{i-1}+1+1\right)=$ $k_{i-2}+2 \rightarrow \infty, b_{T_{l}} \rightarrow c$ as $i \rightarrow \infty$. Furthermore, $\tilde{Q}(l+1) \geq k_{i-1}$, and also $c_{T_{l}} \rightarrow c$ as $i \rightarrow \infty$. Hence $\left|b_{T_{l}}-c_{T_{l}}\right| \rightarrow 0$. 
xix By construction of $Q$, there are no cutting times between $S_{k_{i}+5}$ and $S_{k_{i+1}-1}$. This finishes the proof.

Proof of Example 11.2. First we check the admissibility condition (2.3)

- If $k \equiv 0 \bmod 3$, then $Q(k+1)=k-1>0=Q\left(Q^{2}(k)+1\right)$.

- If $k \equiv 1 \bmod 3$, then $Q(k+1)=k>0=k-3=Q(k-3)=Q\left(Q^{2}(k)+1\right)$.

- If $k \equiv 2 \bmod 3$, then $Q(k+1)=0=Q(k-2)=Q\left(Q^{2}(k)+1\right)$, and $Q(k+2)=k>k-3=Q(k-1)=Q\left(Q^{2}(k)+2\right)$. Hence $Q$ is admissible.

Because $\liminf _{k} Q(k)=0, M_{S_{k}-1}\left(c_{1}\right) \supset\left(z_{0}, c\right)$ or $\left(c, \hat{z}_{0}\right)$ infinitely often. Hence $R_{n}\left(c_{1}\right) \nrightarrow 0$.

Let $U$ some neighbourhood of $c$. We claim that $M_{n}\left(c_{1}\right) \supset U$ at most finitely often. Let $M_{n-1}\left(c_{1}\right)=\left(a_{n}, b_{n}\right)=\left(c_{n-S\langle n\rangle}, c_{n-T\langle n\rangle}\right)$ as in the previous proof.

- If $k \equiv 0 \bmod 3$, then, using (11.3), $T\left\langle S_{k}\right\rangle=T\left\langle S_{k-1}\right\rangle=S_{k-1}-S_{Q^{2}(k-1)}$ and $T_{+}\left\langle S_{k}\right\rangle=S_{k-1}+S_{Q\left(Q^{2}(k-1)+1\right)}+S_{Q\left(Q^{2}(k-1)+2\right)}=S_{k}+S_{k-4}$. Therefore $T_{+}\left\langle S_{k}\right\rangle-S_{k} \rightarrow \infty$ and $\left|b_{S_{k}}-c_{S_{k}}\right| \rightarrow 0$ as $k \rightarrow \infty$. Because $Q(k+1)=k-1 \rightarrow$ $\infty$, also $c_{S_{k}} \rightarrow c$.

- If $k \equiv 1 \bmod 3$, then $T\left\langle S_{k}\right\rangle=S_{k}-S_{Q^{2}(k)}=S_{k}-S_{k-3}$ and $T_{+}\left\langle S_{k}\right\rangle=$ $S_{k}+S_{Q\left(Q^{2}(k)+1\right)}=S_{k}+S_{Q(k-2)}=S_{k}+S_{k-3}$. Therefore $T_{+}\left\langle S_{k}\right\rangle \rightarrow \infty$ and $\left|b_{S_{k}}-c_{S_{k}}\right| \rightarrow 0$ as $k \rightarrow \infty$. Because $Q(k+1)=k \rightarrow \infty$, also $c_{S_{k}} \rightarrow \infty$.

- If $k \equiv 2 \bmod 3$, then $a_{S_{k}}=S_{k-1}$ and $Q(k-1+1)=k-1 \rightarrow \infty$ as $k \rightarrow \infty$. Hence $a_{S_{k}} \rightarrow c$.

Finally, we check the co-cutting times. Let $T_{l}$ be a co-cutting time, and $S_{k}=$ $S_{+}\left\langle T_{l}\right\rangle$. Then $k \equiv 1 \bmod 3$ or $k \equiv 2 \bmod 3$, and in either case $Q^{2}(k)=k-3$. Hence $S_{+}\left\langle T_{l}\right\rangle-T_{l} \geq S_{k-3} \rightarrow \infty$ as $l \rightarrow \infty$. Therefore $a_{T_{l}} \rightarrow c_{T_{l}}$ as $l \rightarrow \infty$. This proves the claim. By the Contraction Principle, it follows that $r_{n}\left(c_{1}\right) \rightarrow 0$. The proof is finished.

Proof of Example 11.3. For the large part we can copy the proof of Example 11.2. This shows that $d\left(c, \partial M_{r}\left(c_{1}\right)\right) \rightarrow 0$ if $r \rightarrow \infty, M_{r}\left(c_{1}\right) \ni c$ and $r \neq S_{4 i+3}$ for some $i$. For $r=S_{k}-1:=S_{4 i+3}-1$, the situation is different: As $Q(k+1)=Q(Q(k)+1)=0$, it follows that $c_{S_{k}} \in A_{0}$ and $a_{S_{k}}=c_{Q(k)} \in \hat{A}_{0}$. Therefore $M_{r}\left(c_{1}\right) \supset\left(z_{0}, \hat{z}_{0}\right)$. Furthermore,

$$
T\left\langle S_{k}\right\rangle=T\left\langle S_{k-1}\right\rangle=S_{k-1}-S_{Q^{2}(k-1)}=S_{k-1}-S_{k-5},
$$

and

$$
\begin{aligned}
T_{+}\left\langle S_{k}\right\rangle & =S_{k-1}+S_{Q\left(Q^{2}(k-1)+1\right)}+S_{Q\left(Q^{2}(k-1)+2\right)}+S_{Q\left(Q^{2}(k-1)+3\right)} \\
& =S_{k}+1+S_{k-5} .
\end{aligned}
$$

Therefore $T_{+}\left\langle S_{k}\right\rangle-S_{k} \rightarrow \infty$ and $d\left(b_{k}, z_{0}\right) \rightarrow 0$. This concludes the proof.

\section{REFERENCES}

[B1] H. Bruin, Topological conditions for the existence of invariant measures for unimodal maps, Ergod. Th. and Dyn. Sys. 14 (1994), 433-451. MR 95m:58086

[B2] H. Bruin, Invariant measures of interval maps, Thesis, Delft. (1994).

[B3] H. Bruin, Combinatorics of the kneading map, Int. Jour. Bif. and Chaos 5 (1995), 13391349. MR 96k:58070

[BKNS] H. Bruin, G. Keller, T. Nowicki, S. van Strien, Wild Cantor attractors exist, Ann. of Math 143 (1996), 97-130. MR 96m:58145

[BL1] A. M. Blokh, M. Lyubich, Attractors of maps of the interval, Banach Center Publ. 23 (1986), 427-442. MR 92k:58068 
[BL2] A.M. Blokh, M. Lyubich, Measurable dynamics of S-unimodal maps of the interval, Ann. Scient. Éc. Norm. Sup. $4^{e}$ série, 24 (1991), 545-573. MR 93f:58132

[G] J. Guckenheimer, Sensitive dependence on initial conditions for unimodal maps, Commun. Math. Phys. 70 (1979), 133-160. MR 82c:58037

[GJ] J. Guckenheimer, S. Johnson, Distortion of S-unimodal maps, Ann. of Math. 132 (1990), 71-130. MR 91g:58157

[Got] W. H. Gottschalk, Orbit-closure decompositions and almost periodic properties, Bull. A.M.S. 50 (1944), 915-919. MR 6:165a

[GT] J.-M. Gambaudo, C. Tresser, A monotonicity property in one dimensional dynamics, Contemp. Math. 135 (1992), 213-222. MR 93i:58087

$[\mathrm{H}] \quad$ F. Hofbauer, The topological entropy of the transformation $x \mapsto a x(1-x)$, Monath. Math. 90 (1980), 117-141. MR 82e:28025

[HK] F. Hofbauer, G. Keller, Some remarks on recent results about S-unimodal maps, Ann. Inst. Henri Poincaré, Physique Théorique 53 (1990), 413-425. MR 92m:58077

[Jo] S. D. Johnson, Singular measures without restrictive intervals, Commun. Math. Phys. 110 (1987), 185-190. MR 88g:58093

[JS1] M. Jakobson, G. Świątek, Metric properties of non-renormalizable S-unimodal maps, Preprint IHES/M/91/16 (1991).

[JS2] M. Jakobson, G. Świątek, Metric properties of non-renormalizable S-unimodal maps. Part I: Induced expansion and invariant measures, Ergod. Th. \& Dyn. Sys. 14 (1994), 721-755. MR 95i:58116

[JS3] M. Jakobson, G. Świạtek, Quasisymmetric conjugacies between unimodal maps, Preprint Stony Brook 16 (1991).

[KN] G. Keller, T. Nowicki, Fibonacci maps re(a))-visited, Ergod. Th. and Dyn. Sys. 15 (1995), 99-120. MR 95k:58098

[L1] M. Lyubich, Combinatorics, geometry and attractors of quasi-quadratic maps, Ann. of Math. 140 (1994), 347-404. MR 95j:58108

[L2] M. Lyubich, Milnor's attractors, persistent recurrence and renormalization, Topological Methods in Modern Mathematics (Stony Brook, NY, 1991; L. R. Goldberg and A. V. Phillips, editors), Publish or Perish, Inc., Houston, TX, 1993, pp. 513-541. MR 94e:58082

[LM] M. Lyubich, J. Milnor, The Fibonacci unimodal map, Journ. A.M.S. 6 (1993), 425-457. MR 93h:58080

[Ma1] M. Martens, Interval Dynamics, Thesis, Delft, 1990.

[Ma2] M. Martens, Distortion results and invariant Cantor sets of unimodal maps, Ergod. Th. and Dyn. Sys. 14 (1994), 331-349. MR 96c:58108

[Mi1] J. Milnor, On the concept of attractor, Commun. Math. Phys. 99 (1985), 177-195; 102 (1985), 517-519. MR 87i:58109a,b

[Mi2] J. Milnor, Local connectivity of Julia sets; Expository lectures, Preprint StonyBrook \# 1992/11.

[MS] W. de Melo, S. van Strien, One-dimensional dynamics, Ergebnisse der Mathematik und ihrer Grenzgebiete (1993), Springer-Verlag, Berlin and New York. MR 95a:58035

[NS] T. Nowicki, S. van Strien, Absolutely continuous invariant measures for $C^{2}$ unimodal maps satisfying the Collet-Eckmann conditions, Invent. Math. 93 (1988), 619-635. MR 89j:58068

Department of Mathematics, Royal Institute of Technology, 10044 Stockholm, SWEDEN

E-mail address: e-mail bruin@math.kth.se 